DESY 08-113

PITHA 08/19

\title{
TESTING THE MAJORANA NATURE OF GLUINOS AND NEUTRALINOS
}

\author{
S. Y. Choi ${ }^{1}$, M. Drees ${ }^{2,3,4}$, A. Freitas ${ }^{5,6}$, and P. M. Zerwas ${ }^{7,8}$ \\ 1 Department of Physics and RIPC, Chonbuk National University, Jeonju 561-756, Korea \\ 2 Physikalisches Inst. der Univ. Bonn, D-53115 Bonn, Germany \\ 3 School of Physics, KIAS, Seoul 130-012, Korea \\ 4 Bethe Center of Theoretical Physics, Univ. Bonn, D-53115 Bonn, Germany \\ 5 Department of Physics 8 Astronomy, University of Pittsburgh, PA 15260, USA \\ ${ }^{6}$ HEP Division, Argonne National Laboratory, Argonne, IL 60439, USA \\ 7 Deutsches Elektronen-Synchrotron DESY, D-22603 Hamburg, Germany \\ 8 Inst. Theor. Physik E, RWTH Aachen U, D-52074 Aachen, Germany
}

(Dated: December 16, 2008)

\begin{abstract}
Gluinos and neutralinos, supersymmetric partners of gluons and neutral electroweak gauge and Higgs bosons, are Majorana particles in the Minimal Supersymmetric Standard Model [MSSM]. Decays of such self-conjugate particles generate charge symmetric ensembles of final states. Moreover, production channels of supersymmetric particles at colliders are characteristically affected by the Majorana nature of particles exchanged in the production processes. The sensitivity to the Majorana character of the particles can be quantified by comparing the predictions with Dirac exchange mechanisms. A consistent framework for introducing gluino and neutralino Dirac fields can be designed by extending the $N=1$ supersymmetry of the $M S S M$ to $N=2$ in the gauge sector. We examine to which extent like-sign dilepton production in the processes $q q \rightarrow \tilde{q} \tilde{q}$ and $e^{-} e^{-} \rightarrow \tilde{e}^{-} \tilde{e}^{-}$is affected by the exchange of either Majorana or Dirac gluinos and neutralinos, respectively, at the Large Hadron Collider (LHC) and in the prospective $e^{-} e^{-}$mode of a lepton linear collider.
\end{abstract}

\section{INTRODUCTION}

In the Minimal Supersymmetric Standard Model [MSSM] gauge super-multiplets are built up by two components, bosonic gauge fields and fermionic gaugino fields, Refs. [1-3]. Since neutral vector fields are self-conjugate, the corresponding supersymmetric partners are Majorana fields. Condensing the gluon fields in the color-octet matrix $g$ and the gluinos in the color-octet matrix $\tilde{g}$, the (color) charge conjugate fields $g^{c}$ and $\tilde{g}^{c}$ are related to the original fields by

$$
\begin{aligned}
& g^{c}=-g^{T} \\
& \tilde{g}^{c}=-\tilde{g}^{T} .
\end{aligned}
$$

For the electroweak gauge and Higgs bosons and the neutralinos, mixtures of fermionic gauginos and higgsinos, analogous relations hold.

The gluino and neutralino Majorana particles carry masses which are rooted in the Higgs and the (soft) supersymmetry breaking sector. Massive Majorana fields can be distinguished experimentally from Dirac fields in gauge theories quite generally. [For massless fields the distinction is more subtle, depending on the form of the interactions in the theory.] In this report we will study the characteristic differences between Majorana and Dirac fields and work out the experimental implications. The analyses will be performed in a hybrid scheme, Ref. [4], in which the minimal $\mathrm{N}=1$ supersymmetric standard model is extended by gauge elements of $\mathrm{N}=2$ supersymmetry [5].

Majorana fields in $\mathrm{N}=1$ supersymmetric theories are characterized by two self-conjugate L- and R-components in 
parallel to the two vector field components. These fermionic components can be paired with two additional fermionic fields in $\mathrm{N}=2$ supersymmetric theories, in which a vector super-multiplet is combined with an additional chiral supermultiplet to a vector hyper-multiplet. If the Majorana masses are identical and the fields are mixed maximally, the four fermionic degrees of freedom can join to a Dirac field and its charge-conjugate companion [6]. In this limit the theory includes vector fields, Dirac gaugino fields and scalars, all states belonging to the adjoint representation of the gauge group.

The four Higgs superfields belong to a chiral and an anti-chiral multiplet. By contrast, the matter superfields sui generis are restricted in the $\mathrm{N}=1 / \mathrm{N}=2$ hybrid scheme to the standard $\mathrm{N}=1$ chiral component in accordance with the experimental fact that matter fermions are chiral.

In this setup the $\mathrm{N}=2$ gauge interactions are an extension of the familiar $\mathrm{N}=1$ gauge interactions. The additional component of the $\mathrm{N}=2$ interaction between the new gaugino field and the Higgs fields can be reinterpreted as component of a superpotential affecting the neutralino and chargino masses after the electroweak symmetry is broken. [In addition the Higgs self-interactions are modified, not affecting the present analysis though.]

Soft supersymmetry breaking gives rise, at the phenomenological level, to three gaugino mass parameters: two Majorana masses $M_{a}$ and $M_{b}$, and a mixing term $M_{a b}$. The first Majorana mass may be associated with the $\mathrm{N}=1$ gaugino mass term, and the second with the new gaugino field. Diagonalizing the $\{a b\}$ mass matrix generates two Majorana masses $m_{1,2}$ and a mixing angle $\theta$, which relates the mass eigenstates to the original current states. Depending on the supersymmetry breaking parameters, $\theta$ can assume any value between 0 and $\pi / 2$. It is easy to design a path in mass-parameter space such that the Dirac limit can be approached smoothly. Tuning the diagonal mass parameters $M_{a, b}$ to zero, only the off-diagonal mixing term $M_{a b}$ survives and, as a result, the mass eigenvalues $m_{1,2}$ become identical, modulo sign, and the mixing of the states maximal, $\theta=\pi / 4$. In the maximal mixing limit the two Majorana states combine into one Dirac fermion (and its antifermion partner). Maximal mixing of Majorana particles guarantees the vanishing of transition amplitudes generally associated with the exchange of Dirac particles.

This procedure is well suited for the strong interaction sector. The electroweak sector is less transparent due to the complicated mixing effects beyond the soft supersymmetry breaking terms after electroweak symmetry breaking. In the limit in which the supersymmetry breaking scale is significantly larger than the electroweak scale, the Dirac limit is approached approximately. Though sounding strange at first glance, it is clear in the light of the previous comments that a quantitative definition can be formulated for the concept of a near-Dirac field or particle.

Adopting this extension of the MSSM to a $\mathrm{N}=1 / \mathrm{N}=2$ hybrid model, observables can be designed for experimental analyses at the LHC [7], which allow us to follow a smooth transition from a Majorana theory of gluinos (and neutralinos) to a Dirac theory. The standard examples are the equal-chirality transition amplitudes

$$
q_{L} q_{L} \rightarrow \tilde{q}_{L} \tilde{q}_{L} \text { and } q_{R} q_{R} \rightarrow \tilde{q}_{R} \tilde{q}_{R} .
$$

These amplitudes are non-zero for Majorana gluino exchange but they vanish for Dirac gluino exchange in the $\mathrm{N}=1 / \mathrm{N}=2$ hybrid theory. [The same arguments can be applied to $e_{L}^{-} e_{L}^{-} \rightarrow \tilde{e}_{L}^{-} \tilde{e}_{L}^{-}$, and $\mathrm{L} \Rightarrow \mathrm{R}$, for electroweak gauginos.] However, amplitudes for the transition from 2 -fermion to 0 -fermion states do not vanish in general. In the present context the mixed-chirality amplitude $q_{L} q_{R} \rightarrow \tilde{q}_{L} \tilde{q}_{R}$ is non-zero for Dirac exchange and, in fact, equal to the amplitude for Majorana exchange [analogously for $e_{L}^{-} e_{R}^{-}$scattering].

Using left/right-handedly polarized beams in the $e^{-} e^{-}$collision mode of a linear collider [8], the rules outlined above can easily be applied for studying the Majorana/Dirac nature of neutralinos experimentally. In addition, it has been demonstrated earlier when discussing potential measurements of the $q \tilde{q} \tilde{g}$ Yukawa coupling, Refs. [9, 10], that the analysis of like-sign dilepton final states in $p p$ collisions at the LHC signals $\tilde{q}_{L} \tilde{q}_{L}$ final states in supersymmetric theories. Adjusting the $\tilde{q}_{L}$ decays to the Dirac limit, the analyses of Refs. $[9,10]$ can be transferred, mutatis mutandis, easily. The potential of like-sign dilepton signatures for discriminating Majorana from Dirac structures of supersymmetric theories has also been noted in Ref. [11]. 


\begin{tabular}{|c||c|c|c|}
\hline Group & Spin 1 & Spin $1 / 2$ & Spin 0 \\
\hline \hline $\mathrm{SU}(3)$ & $g$ & $\tilde{g} ; \tilde{g}^{\prime}$ & $\sigma_{g}$ \\
$\mathrm{SU}(2)$ & $W^{ \pm}, W^{0}$ & $\tilde{W}^{ \pm}, \tilde{W}^{0} ; \tilde{W}^{\prime \pm}, \tilde{W}^{\prime 0}$ & $\sigma_{W}^{ \pm}, \sigma_{W}^{0}$ \\
$\mathrm{U}(1)$ & $B$ & $\tilde{B} ; \tilde{B}^{\prime}$ & $\sigma_{B}$ \\
\hline
\end{tabular}

TABLE I: The $\mathrm{N}=2$ gauge hyper-multiplets.

These processes are complementary to tests of the Majorana nature of gluinos in gluino decays, notably to top plus stop final states, which have been discussed widely in the literature [12]. Moreover, two-gluino final states decaying to bottom + sbottom quarks have served as an important channel for searching for supersymmetry at the Tevatron [13]. Likewise, analyses of like-sign chargino production [14] at the LHC and neutralino decays [15] have been studied extensively in the past for testing the Majorana character of neutralinos.

The report is organized as follows. In the next Section 2 we define the essential elements of the $N=1 / N=2$ hybrid model and establish the phenomenological base. In Sections 3 and 4 we discuss subsequently the strong interaction gluino sector and the electroweak sector, including the concept of a near-Dirac field, first in the limit in which the electroweak breaking scale can be neglected compared to the supersymmetry parameters, and second the systematic approximation to this limit. In Section 5 like-sign dileptons will be analyzed as a signal for the Majorana to Dirac transition at the LHC, before Section 6 concludes this study.

\section{THEORETICAL BASIS: $\mathrm{N}=1 / \mathrm{N}=2$ HYBRID MODEL}

In the MSSM based on $\mathrm{N}=1$ supersymmetry, bosonic gauge fields are one-to-one paired with fermionic spin $1 / 2$ gaugino fields and Higgs bosons with higgsinos. Fermionic lepton and quark matter fields are paired with bosonic spin-0 sleptons and squarks. The neutral gauginos in this ensemble are self-conjugate Majorana fields with two chirality components corresponding to the two helicity states of the gauge fields. Decay channels of these particles and their exchange in production processes generate characteristic signatures of their Majorana nature. The uniqueness of these characteristics can be proven by comparing the signatures with predictions derived from Dirac theories.

It turns out that $\mathrm{N}=2$ supersymmetry offers a theoretically solid platform for a consistent comparison between Majorana and Dirac theories $[4,16]$. The gauge super-multiplets are expanded to hyper-multiplets which incorporate new chiral superfields composed of a gaugino and a scalar field. In the following these new gaugino fields will be labeled by an apostrophe. For the standard $\mathrm{SU}(3) \times \mathrm{SU}(2) \times \mathrm{U}(1)$ gauge group the $\mathrm{N}=2$ fields and their components are summarized in Table I. As argued before, the superposition of two Majorana fields carrying equal masses and being mixed maximally can be reinterpreted as a Dirac field. By tuning the masses of the $\mathrm{N}=1$ gauginos and the new gauginos a path for a continuous transition from a Majorana to a Dirac theory can be designed.

The two disjoint $\mathrm{N}=1$ super-fields of the MSSM Higgs sector $\hat{H}_{d}$ and $\hat{H}_{u}$ can be united in an $\mathrm{N}=2$ hyper-multiplet, composed of $\hat{H}_{d}$ as a chiral field and $\hat{H}_{u}^{\dagger}$ as its anti-chiral companion, cf. Ref. [17].

In a similar way the chiral matter superfields of (s)leptons and (s)quarks, generically called $\hat{Q}$, are extended by new anti-chiral matter fields $\hat{Q}^{\prime}$ to hyper-fields. None of the mirror fields $\hat{Q}^{\prime}$ that include new leptons and quarks has been observed so far. Given the success of the chiral standard theory, either the mirror particles are very heavy, or this component is assumed absent a priori. The second scenario may be realized in $\mathrm{N}=2$ theories including extra space dimensions in which $\mathrm{N}=1$ matter super-fields are restricted only to 4-dimensional branes [18].

Alternative supersymmetric scenarios with Dirac gauginos are based on D-term supersymmetry breaking models [19] or exact continuous R-symmetries [20]. On the phenomenological level these models lead to identical formulations of the Dirac gauginos but it is less straightforward to define a continuous Majorana-Dirac transition. 
In the following we will adopt the $\mathrm{N}=1 / \mathrm{N}=2$ hybrid scenario as the base for phenomenological studies of smooth transitions from Majorana to Dirac fields. The model appears minimal in view of the basic field degrees of freedom and their interactions. For the present purpose there is little difference between the $\mathrm{N}=2$ form of the Higgs sector or two disjoint $\mathrm{N}=1$ Higgs sectors treated in parallel to the matter fields. [An increased mass range of the lightest Higgs boson and additional self-couplings however render the extended option attractive in itself.]

Concentrating on the gaugino sector in regard of the Majorana to Dirac transition, the Lagrangian derived from the general $\mathrm{N}=2$ action can be restricted to a few relevant terms:

\subsection{Hyper-QCD Sector}

Standard gluino $\tilde{g}$ and new gluino $\tilde{g}^{\prime}$ fields are coupled minimally to the gluon field $g$,

$$
\mathcal{L}_{\mathrm{QCD}}^{g \tilde{g} \tilde{g}}=g_{s} \operatorname{Tr}\left(\overline{\tilde{g}} \gamma^{\mu}\left[g_{\mu}, \tilde{g}\right]+\overline{\tilde{g}}^{\prime} \gamma^{\mu}\left[g_{\mu}, \tilde{g}^{\prime}\right]\right)
$$

with the fields condensed to color-octet matrices $g_{\mu}=\frac{1}{\sqrt{2}} \lambda^{a} g_{\mu}^{a}$ etc., $g_{s}$ denoting the QCD coupling, and two 4component Majorana spinor fields $\tilde{g}$ and $\tilde{g}^{\prime}$ satisfying $(\tilde{g})^{c}=-\tilde{g}^{T}$ and $\left(\tilde{g}^{\prime}\right)^{c}=-\tilde{g}^{T}$. The Lagrangian generates the usual $\tilde{g} \tilde{g} g$ and $\tilde{g}^{\prime} \tilde{g}^{\prime} g$ vertices for gluinos coupled to gluons. Matter fields only interact with the standard gluino,

$$
\mathcal{L}_{\mathrm{QCD}}^{q \tilde{q} \tilde{g}}=-g_{s}\left[\overline{q_{L}} \tilde{g} \tilde{q}_{L}-\overline{q_{R}} \tilde{g} \tilde{q}_{R}+\text { h.c. }\right],
$$

while $\mathrm{N}=2$ supersymmetry requires $\tilde{g}^{\prime}$ to only couple to the hyper-multiplet partners of the $\mathrm{N}=1$ quarks/squarks which, in the hybrid theory, are assumed to be projected out. ${ }^{1}$

Soft supersymmetry breaking generates masses for the gluino fields $\tilde{g}$ and $\tilde{g}^{\prime}$. Diagonal terms in the fields $\tilde{g}$ and $\tilde{g}^{\prime}$ generate the individual Majorana mass parameters $M_{3}$ and $M_{3}^{\prime}$ while an off-diagonal term coupling $\tilde{g}$ with $\tilde{g}^{\prime}$ will be crucial for the transition of the two Majorana fields to a joined Dirac field:

$$
\mathcal{L}_{\mathrm{QCD}}^{m}=-\frac{1}{2}\left[M_{3}^{\prime} \operatorname{Tr}\left(\overline{\tilde{g}^{\prime}} \tilde{g}^{\prime}\right)+M_{3} \operatorname{Tr}(\overline{\tilde{g}} \tilde{g})+M_{3}^{D} \operatorname{Tr}\left(\overline{\tilde{g}^{\prime}} \tilde{g}+\overline{\tilde{g}} \tilde{g}^{\prime}\right)\right]
$$

[For the purpose of our analysis, all mass parameters are assumed real throughout the paper.] As worked out in detail in the next section, diagonalizing the $\tilde{g}^{\prime}, \tilde{g}$ mass matrix [in the left-chirality basis, i.e. $\tilde{g}_{L}=\frac{1}{2}\left(1-\gamma_{5}\right) \tilde{g}$ etc.]

$$
\mathcal{M}_{g}=\left(\begin{array}{cc}
M_{3}^{\prime} & M_{3}^{D} \\
M_{3}^{D} & M_{3}
\end{array}\right)
$$

gives rise to two Majorana mass eigenstates, $\tilde{g}_{1}$ and $\tilde{g}_{2}$ with masses $m_{1}$ and $m_{2}$. For large new gluino masses, $M_{3}^{\prime} \rightarrow \pm \infty$, the standard MSSM gluino sector is recovered. On the other side, in the limit in which the Majorana mass parameters $M_{3}$ and $M_{3}^{\prime}$ vanish but the off-diagonal element $M_{3}^{D}$ is non-zero, the mixing between the states is maximal and the two Majorana states, carrying identical masses, can be paired to a Dirac state. Thus varying $M_{3}^{\prime}$ from infinity to zero while trailing $M_{3}$ from a TeV-scale value to zero, a continuous path can be constructed for the transition from the MSSM gluino Majorana theory to a Dirac theory.

Table I shows that the hybrid theory also contains a complex scalar octet $\sigma_{g}$. Its coupling to gluons is determined by $\mathrm{SU}(3)$ gauge invariance. In addition, $\mathrm{N}=2$ supersymmetry stipulates [5] the existence of a $\sigma_{g} \tilde{g} \tilde{g}^{\prime}$ coupling, while

\footnotetext{
1 One could contemplate a non-supersymmetric theory with Dirac gluinos where $\tilde{g}^{\prime} \bar{q} \tilde{q}$ couplings exist. This would tend to increase the differences between Majorana and Dirac gluinos, e.g. leading to different total cross sections for associate $g q \rightarrow \tilde{q} \tilde{g}$ production. The $\mathrm{N}=1 / \mathrm{N}=2$ hybrid analyzed by us is better motivated; considering it as alternative of the usual MSSM is also conservative in the sense that it minimizes the differences.
} 
the couplings of $\sigma_{g}$ to quarks also involve their hyper-multiplet partners. The hybrid theory predicts pair production of $\sigma_{g}$ scalars. However, this is not directly related to the Dirac or Majorana nature of the gluinos, which is the central issue of our analysis. The detailed phenomenology of the new scalars will be described in a sequel to this report.

\subsection{Electroweak Sector}

The electroweak neutralino/chargino sector is considerably more complicated than the QCD sector due to the mixing of gauginos and higgsinos induced by electroweak symmetry breaking. The complexity increases only slightly in the extension from $\mathrm{N}=1$ to $\mathrm{N}=2$ supersymmetry. While the expansion of the $\tilde{W}, \tilde{B}$ isospin and hypercharge sector by the $\tilde{W}^{\prime}, \tilde{B}^{\prime}$ fields runs strictly parallel to the gluino sector, the embedding of the Higgs fields into a chiral and antichiral $\mathrm{N}=2$ hyper-multiplet generates new gauge interactions which couple the Higgs super-fields with the new chiral superfields of the $\mathrm{N}=2$ vector multiplets:

$$
W_{\text {higge }^{\text {gauge' }}}^{\text {f }^{\prime}}=\sqrt{2} g \hat{H}_{u} \cdot\left(I^{a} \hat{H}_{d}\right) \hat{W}^{\prime a}+\sqrt{2} g^{\prime} \hat{H}_{u} \cdot\left(Y \hat{H}_{d}\right) \hat{B}^{\prime},
$$

where $I^{a}=\tau^{a} / 2(a=1,2,3)$ and $Y$ are the weak isospin and hypercharge generators, respectively, $g$ and $g^{\prime}$ are the $\mathrm{SU}(2)$ and $\mathrm{U}(1)_{\mathrm{Y}}$ gauge couplings, and the central dot denotes an $\mathrm{SU}(2)$-invariant contraction. The $\mathrm{N}=2$ supersymmetry allows for a bilinear $\mu$ Higgs/higgsino coupling,

$$
W_{\text {higgs }}^{\text {bilin }}=\mu \hat{H}_{u} \cdot \hat{H}_{d}
$$

[in the standard notation with the $\mathrm{SU}(2)$-invariant contraction $\hat{H}_{u} \cdot \hat{H}_{d}=\hat{H}_{u}^{+} \hat{H}_{d}^{-}-\hat{H}_{u}^{0} \hat{H}_{d}^{0}$, etc].

The additional gauge-strength Yukawa interactions,

$$
\mathcal{L}_{\text {higgs }^{\text {gauge }}}^{{ }^{\prime}}=-\frac{g}{\sqrt{2}}\left[H_{u} \cdot\left(\tau^{a} \tilde{H}_{d}\right) \tilde{W}^{\prime a}+H_{d} \cdot\left(\tau^{a} \tilde{H}_{u}\right) \tilde{W}^{\prime a}\right]-\frac{g^{\prime}}{\sqrt{2}}\left[H_{d} \cdot \tilde{H}_{u} \tilde{B}^{\prime}-H_{u} \cdot \tilde{H}_{d} \tilde{B}^{\prime}\right],
$$

generated from the superpotential Eq. (2.5), lead, after electroweak symmetry breaking,

$$
\begin{array}{ll}
\text { Neutralinos: } & \mathcal{L}_{\text {higgs }}^{\chi^{\prime 0}}=-m_{Z}\left[s_{W}\left(s_{\beta}{\tilde{\tilde{B}_{R}^{\prime}}}_{H_{d L}}^{0}+c_{\beta} \overline{\tilde{H}_{u R}^{0}} \tilde{B}_{L}^{\prime}\right)-c_{W}\left(c_{\beta} \overline{\tilde{H}_{u R}^{0}} \tilde{W}_{L}^{\prime 0}+s_{\beta} \overline{\tilde{W}_{R}^{\prime 0}} \tilde{H}_{d L}^{0}\right)+\text { h.c. }\right], \\
\text { Charginos: } & \mathcal{L}_{\text {higgs }}^{\chi^{\prime \pm}}=-\sqrt{2} m_{W} c_{\beta} \overline{\tilde{H}_{u R}^{-}} \tilde{W}_{L}^{\prime-}+\sqrt{2} m_{W} s_{\beta} \overline{\tilde{W}_{R}^{\prime-}} \tilde{H}_{d L}^{-}+\text {h.c. },
\end{array}
$$

to off-diagonal mass terms and mixings between the standard higgsinos and the new winos $\tilde{W}^{\prime}$ and bino $\tilde{B}^{\prime}$. Choosing the left-chirality bases $\left\{\tilde{B}^{\prime 0}, \tilde{B}^{0}, \tilde{W}^{\prime 0}, \tilde{W}^{0}, \tilde{H}_{d}^{0}, \tilde{H}_{u}^{0}\right\}$ and $\left\{\tilde{W}^{\prime \mp}, \tilde{W}^{\mp}, \tilde{H}_{d, u}^{\mp}\right\}$, the neutralino and chargino mass matrices can be cast into the form

$$
\begin{aligned}
\mathcal{M}_{n}= & \left(\begin{array}{cccccc}
M_{1}^{\prime} & M_{1}^{D} & 0 & 0 & m_{Z} s_{W} s_{\beta} & m_{Z} s_{W} c_{\beta} \\
M_{1}^{D} & M_{1} & 0 & 0 & -m_{Z} s_{W} c_{\beta} & m_{Z} s_{W} s_{\beta} \\
0 & 0 & M_{2}^{\prime} & M_{2}^{D} & -m_{Z} c_{W} s_{\beta} & -m_{Z} c_{W} c_{\beta} \\
0 & 0 & M_{2}^{D} & M_{2} & m_{Z} c_{W} c_{\beta} & -m_{Z} c_{W} s_{\beta} \\
m_{Z} s_{W} s_{\beta} & -m_{Z} s_{W} c_{\beta} & -m_{Z} c_{W} s_{\beta} & m_{Z} c_{W} c_{\beta} & 0 & -\mu \\
m_{Z} s_{W} c_{\beta} & m_{Z} s_{W} s_{\beta} & -m_{Z} c_{W} c_{\beta} & -m_{Z} c_{W} s_{\beta} & -\mu & 0
\end{array}\right), \\
\mathcal{M}_{c} & =\left(\begin{array}{ccc}
M_{2}^{\prime} & M_{2}^{D} & -\sqrt{2} m_{W} \sin \beta \\
M_{2}^{D} & M_{2} & \sqrt{2} m_{W} \cos \beta \\
\sqrt{2} m_{W} \cos \beta & \sqrt{2} m_{W} \sin \beta & \mu
\end{array}\right),
\end{aligned}
$$

with the usual abbreviations $s_{W}=\sin \theta_{W}, s_{\beta}=\sin \beta$, etc. for the electroweak mixing angle $\theta_{W}$ and the SUSY Higgs-Goldstone mixing angle $\beta$. [Evidently, the new $\mathrm{N}=2$ Higgs-gauge interactions (2.5) have little impact on the 
overall structure of the mass matrices. If the Higgs sector is reduced to the standard twin of $\mathrm{N}=1$ Higgs fields, the terms corresponding to Eq.(2.8) are simply reduced to zero.]

The gaugino-gauge interactions are extended analogously to the gluino-gluon sector in Eq. (2.1). A new set of interactions between Higgs, higgsino, gauge and gaugino fields is generated by the $\mathrm{N}=2$ gauge interactions in the Higgs sector, cf. Eq.(2.5).

The superpotential involving matter and Higgs superfields of the hybrid model will be taken over from $\mathrm{N}=1$ supersymmetry, analogously the corresponding soft supersymmetry breaking interactions.

\section{THE GLUINO SECTOR IN SUPER- AND HYPER-QCD}

In the previous section we have derived the mass matrix in the gluino sector of the two Majorana fields $\tilde{g}$ and $\tilde{g}^{\prime}$ in $\mathrm{N}=2$ supersymmetry. In the present section we will determine the mass eigenvalues and the corresponding gluino fields. Two limiting cases of the general softly broken $\mathrm{N}=2$ theory are of particular interest. If one of the Majorana mass parameters in the gluino mass matrix is driven to infinity, we will recover the standard $\mathrm{N}=1$ supersymmetry. On the other hand, if both diagonal mass parameters are chosen zero, the two Majorana fields can be united to a Dirac field. This transition restricts considerably the non-zero scattering amplitudes generated by gluino exchanges. Thus by tuning the mass parameters, a common platform for Majorana and Dirac theories can be built, allowing for continuous transitions between the two types of fields and a proper definition of a "near-Dirac" field.

\subsection{Diagonalization of the $2 \times 2$ Hyper-Gluino Mass Matrix}

For real values of $M_{3}, M_{3}^{\prime}$ and $M_{3}^{D}$ the gluino mass matrix in Eq. (2.4) can be diagonalized by means of the unitary transformation matrix $\mathcal{U}$

$$
\mathcal{U}^{T} \mathcal{M}_{g} \mathcal{U}=\operatorname{diag}\left(m_{\tilde{g}_{1}}, m_{\tilde{g}_{2}}\right) \quad \text { with } \quad \mathcal{U}=\left(\begin{array}{cc}
\cos \theta_{3} & \epsilon_{3} \sin \theta_{3} \\
-\epsilon_{3} \sin \theta_{3} & \cos \theta_{3}
\end{array}\right)\left(\begin{array}{cc}
\eta_{1} & 0 \\
0 & \eta_{2}
\end{array}\right),
$$

where the rotation angle varies between $0 \leq \theta_{3} \leq \pi / 2$ and $\eta_{1,2}$ denote the two Majorana-type phases. The mass eigenvalues read:

$$
m_{\tilde{g}_{1,2}}=\frac{1}{2}|| M_{3}^{\prime}+M_{3}\left|\mp \Delta_{3}\right| \quad \text { with } \quad \Delta_{3}=\sqrt{\left(M_{3}^{\prime}-M_{3}\right)^{2}+4\left(M_{3}^{D}\right)^{2}},
$$

with the ordering $m_{\tilde{g}_{1}} \leq m_{\tilde{g}_{2}}$ by definition. The mixing angle $\theta_{3}$, the sign parameter $\epsilon_{3}$ and the two Majorana-type phases $\eta_{1,2}$ defining the diagonalization matrix $\mathcal{U}$ are given by

$$
\begin{aligned}
& \cos \theta_{3} / \sin \theta_{3}=\sqrt{\frac{1 \pm \epsilon_{3}^{\prime}\left|M_{3}-M_{3}^{\prime}\right| / \Delta_{3}}{2}} \\
& \epsilon_{3}=\operatorname{sign}\left[M_{3}^{D}\left(M_{3}^{\prime}-M_{3}\right)\right] \text { and } \epsilon_{3}^{\prime}=\operatorname{sign}\left[M_{3}^{2}-M_{3}^{\prime 2}\right],
\end{aligned}
$$

and

$$
\begin{array}{lll}
\eta_{1}=1 / i & \text { for } & \operatorname{sign}\left[\operatorname{det}\left(\mathcal{M}_{g}\right) \cdot \operatorname{Tr}\left(\mathcal{M}_{g}\right)\right]=+/-, \\
\eta_{2}=1 / i & \text { for } & \operatorname{sign}\left[\operatorname{Tr}\left(\mathcal{M}_{g}\right)\right]=+/-
\end{array}
$$

[the overall signs of $\eta_{1,2}$ are indeterminate]. The form of the diagonalization matrix $\mathcal{U}$ in Eq. (3.1) guarantees the positivity of the mass eigenvalues $m_{\tilde{g}_{1,2}}$ of the fields

$$
\left(\begin{array}{c}
\tilde{g}_{1 R} \\
\tilde{g}_{2 R}
\end{array}\right)=\mathcal{U}^{T}\left(\begin{array}{c}
\tilde{g}_{R}^{\prime} \\
\tilde{g}_{R}
\end{array}\right) \text { and }\left(\begin{array}{c}
\tilde{g}_{1 L} \\
\tilde{g}_{2 L}
\end{array}\right)=\mathcal{U}^{\dagger}\left(\begin{array}{c}
\tilde{g}_{L}^{\prime} \\
\tilde{g}_{L}
\end{array}\right) \text {. }
$$




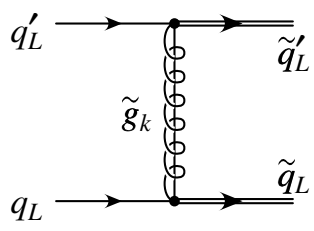

(a)

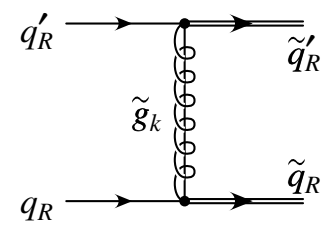

(b)

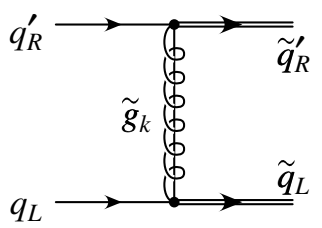

(c)

FIG. 1: Feynman diagrams for squark production of different flavors at hadron colliders. [The index $k$ counts the two gluinos in the $\mathrm{N}=2$ hybrid model, to be ignored for the $\mathrm{N}=1 \mathrm{MSSM}$.]

The rotation by means of the orthogonal sub-matrix of $\mathcal{U}$, combined with the diagonal phase matrix, preserves the Majorana character of the fields $\tilde{g}_{1,2}$. Left- and right-chiral fields are related by charge conjugation: $\left(\tilde{g}_{L}\right)^{c}=-\tilde{g}_{R}^{T}$ and $\left(\tilde{g}_{L}^{\prime}\right)^{c}=-\tilde{g}_{R}^{\prime T}$.

\subsection{Chiral Transition Amplitudes in the Hybrid Model}

The most transparent example for studying the Majorana/Dirac nature of gluinos is the transition between pairs of quarks to pairs of squarks with different flavor:

$$
\begin{aligned}
q_{L} q_{L}^{\prime} & \rightarrow \tilde{q}_{L} \tilde{q}_{L}^{\prime}, \quad q_{R} q_{R}^{\prime} \rightarrow \tilde{q}_{R} \tilde{q}_{R}^{\prime}, \\
q_{L} q_{R}^{\prime} & \rightarrow \tilde{q}_{L} \tilde{q}_{R}^{\prime},
\end{aligned}
$$

The corresponding Feynman diagrams are depicted in Fig. 1. The kernels of the transition matrix elements, involving the $t$-channel exchange of the two Majorana gluinos $\tilde{g}_{k}$, can be cast into the form:

$$
\begin{aligned}
A\left[q_{L} q_{L}^{\prime} \rightarrow \tilde{q}_{L} \tilde{q}_{L}^{\prime}\right] & =-\frac{g_{s}^{2}}{2} \sum_{k=1}^{2}\left[\mathcal{U}_{2 k} \mathcal{U}_{2 k}\right] \frac{\left\langle m_{\tilde{g}_{k}}\right\rangle}{t-m_{\tilde{g}_{k}}^{2}}, \\
A\left[q_{R} q_{R}^{\prime} \rightarrow \tilde{q}_{R} \tilde{q}_{R}^{\prime}\right] & =-\frac{g_{s}^{2}}{2} \sum_{k=1}^{2}\left[\mathcal{U}_{2 k}^{*} \mathcal{U}_{2 k}^{*}\right] \frac{\left\langle m_{\tilde{g}_{k}}\right\rangle}{t-m_{\tilde{g}_{k}}^{2}}, \\
A\left[q_{L} q_{R}^{\prime} \rightarrow \tilde{q}_{L} \tilde{q}_{R}^{\prime}\right] & =+\frac{g_{s}^{2}}{2} \sum_{k=1}^{2}\left[\mathcal{U}_{2 k} \mathcal{U}_{2 k}^{*}\right] \frac{\langle q\rangle}{t-m_{\tilde{g}_{k}}^{2}},
\end{aligned}
$$

to be sandwiched between the quark spinors $\bar{v}_{L, R}^{\prime}$ and $u_{L, R} ; t=q^{2}$ denotes the square of the momentum $q$ flowing through the gluino line. The form of these transition amplitudes can easily be traced back to the rules introduced in the previous section. Currents of equal-sign chirality, LL and RR, are coupled by the mass term of the gluino propagator, while currents of opposite-sign chirality, LR, are coupled by the kinetic term $q$.

From the transition amplitudes (3.7) the cross sections can easily be derived as

$$
\begin{aligned}
& \sigma\left[q q^{\prime} \rightarrow \tilde{q}_{L} \tilde{q}_{L}^{\prime}\right]= \sigma\left[q q^{\prime} \rightarrow \tilde{q}_{R} \tilde{q}_{R}^{\prime}\right] \\
&=\frac{2 \pi \alpha_{s}^{2}}{9 s}\left[\frac{c_{3}^{4} s \beta m_{\tilde{g}_{1}}^{2}}{s m_{\tilde{g}_{1}}^{2}+\left(m_{\tilde{g}_{1}}^{2}-m_{\tilde{q}}^{2}\right)^{2}}+\frac{s_{3}^{4} s \beta m_{\tilde{g}_{2}}^{2}}{s m_{\tilde{g}_{2}}^{2}+\left(m_{\tilde{g}_{2}}^{2}-m_{\tilde{q}}^{2}\right)^{2}}+\frac{2 c_{3}^{2} s_{3}^{2} m_{\tilde{g}_{1}} m_{\tilde{g}_{2}}}{m_{\tilde{g}_{1}}^{2}-m_{\tilde{g}_{2}}^{2}}\left(L_{1}-L_{2}\right)\right] \\
& \sigma\left[q q^{\prime} \rightarrow \tilde{q}_{L} \tilde{q}_{R}^{\prime}\right]=\frac{2 \pi \alpha_{s}^{2}}{9 s}\left[c_{3}^{4}\left(\left(1+\frac{2}{s}\left(m_{\tilde{g}_{1}}^{2}-m_{\tilde{q}}^{2}\right)\right) L_{1}-2 \beta\right)+s_{3}^{4}\left(\left(1+\frac{2}{s}\left(m_{\tilde{g}_{2}}^{2}-m_{\tilde{q}^{2}}^{2}\right)\right) L_{2}-2 \beta\right)\right. \\
&\left.+2 c_{3}^{2} s_{3}^{2}\left(\frac{\left(s m_{\tilde{g}_{1}}^{2}+\left(m_{\tilde{g}_{1}}^{2}-m_{\tilde{q}}^{2}\right)^{2}\right) L_{1}-\left(s m_{\tilde{g}_{2}}^{2}+\left(m_{\tilde{g}_{2}}^{2}-m_{\tilde{q}}^{2}\right)^{2}\right) L_{2}}{m_{\tilde{g}_{1}}^{2}-m_{\tilde{g}_{2}}^{2}}-\beta\right)\right]
\end{aligned}
$$


where

$$
L_{k}=\log \frac{(1+\beta)+2\left(m_{\tilde{g}_{k}}^{2}-m_{\tilde{q}}^{2}\right) / s}{(1-\beta)+2\left(m_{\tilde{g}_{k}}^{2}-2 m_{\tilde{q}}^{2}\right) / s}
$$

and $s_{3}=\sin \theta_{3}, c_{3}=\cos \theta_{3}$ and $\beta=\left(1-4 m_{\tilde{q}}^{2} / s\right)^{1 / 2}$; it has been assumed that all squarks have the same mass $m_{\tilde{q}}$. In the next subsection the characteristics of the transition amplitudes will be analyzed in detail.

\subsection{Majorana to Dirac Path in the Hybrid Model}

The $\mathrm{N}=2$ gluino mass matrix $\mathcal{M}_{g}$ is defined by three parameters, two on-diagonal Majorana mass parameters and the off-diagonal mass parameter which couples the two $\mathrm{N}=1$ sectors of the gluino hyper-multiplet. In the physical basis they manifest themselves as two Majorana mass eigenvalues $m_{\tilde{g}_{1,2}}$ and the rotation angle $\theta_{3}$ between the current and mass eigenstates.

If the new gluino mass parameter $M_{3}^{\prime}$ is chosen infinitely large, the hyper-system is reduced effectively to the original $\mathrm{N}=1$ gluon-gluino super-multiplet with the gluino mass determined by $M_{3}$,

$$
\begin{aligned}
m_{\tilde{g}_{1}} \simeq\left|M_{3}-\left(M_{3}^{D}\right)^{2} / M_{3}^{\prime}\right| & \rightarrow\left|M_{3}\right| \\
m_{\tilde{g}_{2}} \simeq\left|M_{3}^{\prime}\right| & \rightarrow \infty,
\end{aligned}
$$

in analogy to the seesaw formula.

The path from the N=1 Majorana theory to the Dirac theory may be defined in such a way that the mass of the lightest gluino is kept fixed. In addition, we may identify the off-diagonal mass parameter $M_{3}^{D}$ with $m_{\tilde{g}_{1}}$ to reduce the number of free parameters. Starting from the Majorana theory, we follow the path

$$
\begin{aligned}
\mathcal{P}: & M_{3}=m_{\tilde{g}_{1}} M_{3}^{\prime} /\left(M_{3}^{\prime}-m_{\tilde{g}_{1}}\right) \quad \text { for } \quad-\infty \leq M_{3}^{\prime} \leq 0 \\
M_{3}^{D} & =m_{\tilde{g}_{1}} .
\end{aligned}
$$

The heavy gluino mass is trailed along according to

$$
m_{\tilde{g}_{2}}=-M_{3}^{\prime}-m_{\tilde{g}_{1}}^{2} /\left(M_{3}^{\prime}-m_{\tilde{g}_{1}}\right)
$$

while the mixing parameters follow from

$$
\cos \theta_{3}=\frac{1}{\sqrt{1+\left(1-M_{3}^{\prime} / m_{\tilde{g}_{1}}\right)^{2}}}, \quad \sin \theta_{3}=\frac{1-M_{3}^{\prime} / m_{\tilde{g}_{1}}}{\sqrt{1+\left(1-M_{3}^{\prime} / m_{\tilde{g}_{1}}\right)^{2}}} .
$$

The path $\mathcal{P}$ can be mapped onto a unit interval by the transformation

$$
M_{3}^{\prime}=m_{\tilde{g}_{1}} \frac{y}{1+y} \quad \text { for } \quad-1 \leq y \leq 0
$$

leading to

$$
m_{\tilde{g}_{2}}=m_{\tilde{g}_{1}}\left(y+\frac{1}{1+y}\right), \quad \text { and } \quad \cos \theta_{3}=\frac{1+y}{\sqrt{1+(1+y)^{2}}}, \quad \sin \theta_{3}=\frac{1}{\sqrt{1+(1+y)^{2}}} .
$$

The transition of the parameters $M_{3} / M_{3}^{\prime}$ and $\sin \theta_{3} / \cos \theta_{3}$ as well as $m_{\tilde{g}_{2}}$ is exemplified in Fig. 2 .

For $y=-1$, corresponding to $M_{3}^{\prime} \rightarrow-\infty$, the Majorana limit for $\mathrm{N}=1$ gluinos is reproduced with the physical mass $m_{\tilde{g}_{1}}$ while the second set of Majorana particles with $m_{\tilde{g}_{2}} \rightarrow \infty$ is removed from the system. 

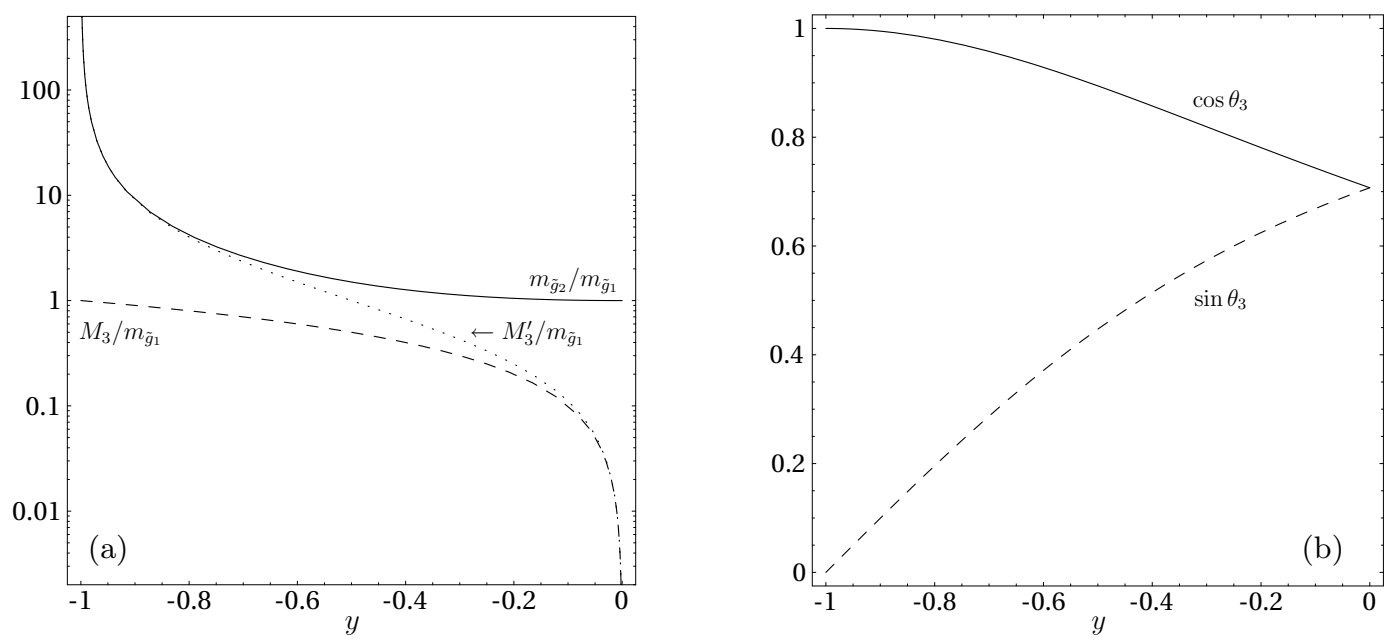

FIG. 2: Illustration of the smooth transition from Majorana $(y=-1)$ to Dirac $(y=0)$ gluino masses (a) and mixing angles (b).

In the right-most end-point $y=0$ of the path $\mathcal{P}$ the Dirac limit is realized. The on-diagonal mass parameters both vanish while the off-diagonal elements are equal:

$$
\begin{aligned}
& M_{3}^{\prime}=M_{3}=0 \text { and } M_{3}^{D} \neq 0 \\
& \sin \theta_{3}=\cos \theta_{3}=1 / \sqrt{2}
\end{aligned}
$$

so that the physical masses $m_{\tilde{g}_{1,2}}=m_{\tilde{g}}$ are identical and the mixing is maximal.

\subsection{The Dirac Limit}

The transformation matrix $\mathcal{U}$, connecting the field bases $\tilde{g} / \tilde{g}^{\prime}$ with $\tilde{g}_{1} / \tilde{g}_{2}$, simplifies in the Dirac limit ${ }^{2}$ to

$$
\begin{aligned}
\mathcal{U} & =\left(\begin{array}{cc}
\cos \theta_{3} & -\sin \theta_{3} \\
\sin \theta_{3} & \cos \theta_{3}
\end{array}\right) \cdot\left(\begin{array}{ll}
1 & 0 \\
0 & i
\end{array}\right)=\left(\begin{array}{cc}
\cos \theta_{3} & -i \sin \theta_{3} \\
\sin \theta_{3} & i \cos \theta_{3}
\end{array}\right) \\
& \rightarrow \frac{1}{\sqrt{2}}\left(\begin{array}{cc}
1 & -1 \\
1 & 1
\end{array}\right) \cdot\left(\begin{array}{ll}
1 & 0 \\
0 & i
\end{array}\right)=\frac{1}{\sqrt{2}}\left(\begin{array}{cc}
1 & -i \\
1 & i
\end{array}\right),
\end{aligned}
$$

corresponding to a $\pi / 4$ rotation matrix and a phase matrix which turns the second eigenvalue positive. Hence the two degenerate physical Majorana fields $\tilde{g}_{1}$ and $\tilde{g}_{2}$ can be expressed in terms of the original current fields $\tilde{g}^{\prime}$ and $\tilde{g}$ as

$$
\begin{aligned}
& \tilde{g}_{1}=\left[\left(\tilde{g}_{L}^{\prime}+\tilde{g}_{R}^{\prime}\right)+\left(\tilde{g}_{L}+\tilde{g}_{R}\right)\right] / \sqrt{2} \\
& \tilde{g}_{2}=i\left[\left(\tilde{g}_{L}^{\prime}-\tilde{g}_{R}^{\prime}\right)-\left(\tilde{g}_{L}-\tilde{g}_{R}\right)\right] / \sqrt{2} .
\end{aligned}
$$

These two Majorana fields are odd and even under charge conjugation: $\tilde{g}_{1}^{c}=-\tilde{g}_{1}^{T}$ and $\tilde{g}_{2}^{c}=+\tilde{g}_{2}^{T}$, respectively.

In this configuration the $t$-channel exchange of the two Majorana fields $\tilde{g}_{1,2}$ in the processes $q_{L} q_{L}^{\prime} \rightarrow \tilde{q}_{L} \tilde{q}_{L}^{\prime}$ and

\footnotetext{
${ }^{2}$ In this degenerate case the mixing matrix $\mathcal{U}$ is unique up to multiplication on the right by an arbitrary orthogonal matrix [21].
} 
$q_{R} q_{R}^{\prime} \rightarrow \tilde{q}_{R} \tilde{q}_{R}^{\prime}$ is maximally destructive and the two amplitudes vanish:

$$
\begin{aligned}
A\left[q_{L} q_{L}^{\prime} \rightarrow \tilde{q}_{L} \tilde{q}_{L}^{\prime}\right] & =A^{*}\left[q_{R} q_{R}^{\prime} \rightarrow \tilde{q}_{R} \tilde{q}_{R}^{\prime}\right] \\
& \sim \sum_{k=1}^{2} \mathcal{U}_{2 k} \mathcal{U}_{2 k}=0 .
\end{aligned}
$$

The superposition of mixed chiral amplitudes, on the other side, is maximally constructive:

$$
A\left[q_{L} q_{R}^{\prime} \rightarrow \tilde{q}_{L} \tilde{q}_{R}^{\prime}\right] \sim \sum_{k=1}^{2} \mathcal{U}_{2 k} \mathcal{U}_{2 k}^{*}=1 .
$$

This picture can be simplified considerably by switching from the two-Majorana to the Dirac description.

Introducing a superposition of two equal-mass Majorana fields, $m_{\tilde{g}_{1}}=m_{\tilde{g}_{2}}=m_{\tilde{g}}$, the right- and left-handed components of the Dirac gluino field coupled to the quark current are effectively given by

$$
\begin{array}{cl}
\tilde{g}_{D}=\left(\tilde{g}_{1}-i \tilde{g}_{2}\right) / \sqrt{2}: & \tilde{g}_{D R}=\tilde{g}_{R} \\
& \tilde{g}_{D L}=\tilde{g}_{L}^{\prime}, \\
\tilde{g}_{D}^{c T}=-\left(\tilde{g}_{1}+i \tilde{g}_{2}\right) / \sqrt{2} \quad: \quad & \tilde{g}_{D R}^{c}=-\tilde{g}_{R}^{\prime T} \\
& \tilde{g}_{D L}^{c}=-\tilde{g}_{L}^{T} .
\end{array}
$$

The field $\tilde{g}_{D}=\left(\tilde{g}_{1}-i \tilde{g}_{2}\right) / \sqrt{2}=\tilde{g}_{R}+\tilde{g}_{L}^{\prime}$ is a Dirac field, i.e. it is not self-conjugate: $\tilde{g}_{D}^{c} \neq \pm \tilde{g}_{D}^{T}$. It describes four degrees of freedom, the two helicities and the particle/antiparticle characteristics. The contraction of the field with itself vanishes [in contrast to Majorana fields], while the contraction between the field and its conjugate is given by the canonical Dirac value. As a result, this Dirac field cannot be exchanged between two chirality-L currents and the LL-type amplitude vanishes. Similarly, the related $\mathcal{C}$-conjugate field $\tilde{g}_{D}^{c}$ is coupled to R-type currents but RR amplitudes vanish. On the other hand, RL-type amplitudes do not vanish and, in fact, the contraction between $\tilde{g}_{D}$ and $\tilde{g}_{D}^{c}$ generates the usual Dirac propagator, so that the RL amplitude corresponds to the standard Dirac exchange amplitude. In summa, the theory of two mass-degenerate Majorana fields with chiral couplings is equivalent to the Dirac theory of a single fermion.

The Lagrangian for the super-QCD interaction of gluinos with squarks and quarks is of the standard N=1 SUSY form (2.2) for one Majorana gluino mass eigenstate $\tilde{g}$ in the Majorana limit, but the interaction Lagrangian of the two Majorana gluino fields in the $\mathrm{N}=1 / \mathrm{N}=2$ hybrid model can be contracted in the Dirac limit to

$$
\begin{aligned}
\mathcal{L}_{\mathrm{QCD}}^{q \tilde{q} \tilde{g}} & =-g_{s} \frac{1}{\sqrt{2}}\left[\overline{q_{L}} \tilde{g}_{1} \tilde{q}_{L}-\overline{q_{R}} \tilde{g}_{1} \tilde{q}_{R}-i\left(\overline{q_{L}} \tilde{g}_{2} \tilde{q}_{L}+\overline{q_{R}} \tilde{g}_{2} \tilde{q}_{R}\right)+\text { h.c. }\right] \\
& =-g_{s}\left[\overline{q_{L}} \tilde{g}_{D} \tilde{q}_{L}+\overline{q_{R}} \tilde{g}_{D}^{c T} \tilde{q}_{R}+\text { h.c. }\right] .
\end{aligned}
$$

The trilinear gluon/gluino interaction is just the sum of the two individual standard interactions. The mass term of the Lagrangian in the Majorana limit,

$$
\mathcal{L}_{\mathrm{QCD}}^{m}=-\frac{1}{2} m_{\tilde{g}} \operatorname{Tr}[\overline{\tilde{g}} \tilde{g}]
$$

is altered in the Dirac limit to

$$
\begin{aligned}
\mathcal{L}_{\mathrm{QCD}}^{m} & =-\frac{1}{2} m_{\tilde{g}} \operatorname{Tr}\left[\overline{\tilde{g}}_{1} \tilde{g}_{1}+\overline{\tilde{g}}_{2} \tilde{g}_{2}\right] \\
& =-m_{\tilde{g}} \operatorname{Tr}\left[\overline{\tilde{g}}_{D} \tilde{g}_{D}\right]
\end{aligned}
$$

in terms of the two degenerate Majorana mass eigenstates, $\tilde{g}_{1}, \tilde{g}_{2}$, and the Dirac field $\tilde{g}_{D}$ or $\tilde{g}_{D}^{c}$, respectively.

As will be demonstrated later in several examples, the transition from the Lagrangian of the 2-Majorana theory to the Dirac theory entails the isomorphism of the two theories in all dynamical aspects, including the (properly defined sets of) cross sections. 


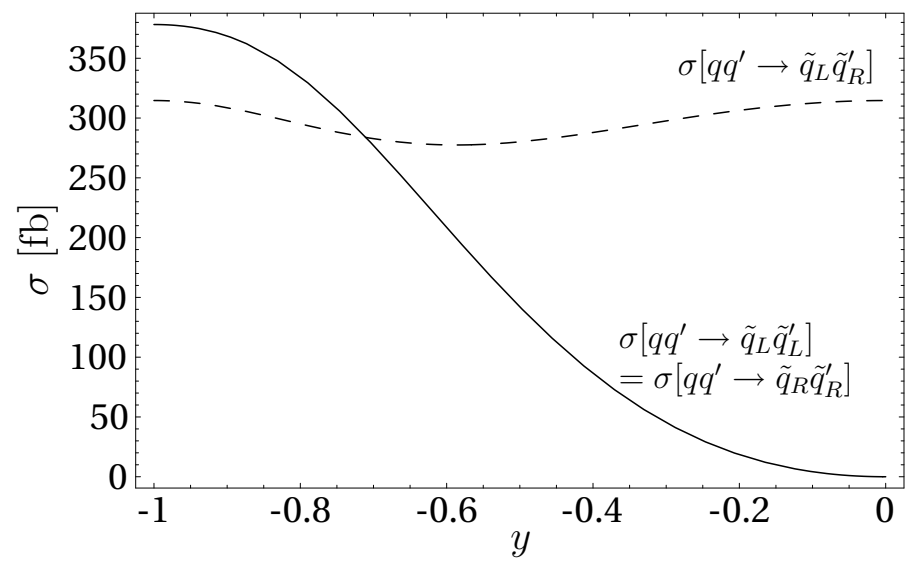

FIG. 3: Partonic cross sections for different-flavor squark production as a function of the Dirac/Majorana control parameter $y$. The plot corresponds to a fixed partonic center-of-mass energy $\sqrt{s}=2000 \mathrm{GeV}$, and $m_{\tilde{q}}=500 \mathrm{GeV}$ and $m_{\tilde{g}_{1}}=600 \mathrm{GeV}$. The scale of the running coupling $\alpha_{s}^{\overline{\mathrm{MS}}}(\mu)$ has been chosen to be $\mu=\sqrt{s}$.

In the Dirac theory, a conserved quantum number $D$, associated with the R-symmetry of the $\mathrm{N}=2$ theory noted in Ref. [11], can be assigned to each supersymmetric particle state appearing in the Lagrangian, nota bene the interaction term (3.23):

$$
\begin{aligned}
& D\left[\tilde{q}_{L}\right]=D\left[\tilde{g}_{D}^{c}\right]=D\left[\tilde{l}_{L}^{-}\right]=D\left[\tilde{\chi}_{D}^{c 0}\right]=D\left[\tilde{\chi}_{D 1}^{+}\right]=-1 \\
& D\left[\tilde{q}_{R}\right]=D\left[\tilde{g}_{D}\right]=D\left[\tilde{l}_{R}^{-}\right]=D\left[\tilde{\chi}_{D}^{0}\right]=D\left[\tilde{\chi}_{D 2}^{+}\right]=+1 .
\end{aligned}
$$

The $\tilde{\chi}_{D}^{ \pm}$components relevant for the gauge-strength Yukawa interactions are $\tilde{\chi}_{D 1 R}^{-}=\tilde{W}_{R}^{-}$and $\tilde{\chi}_{D 2 R}^{+}=\tilde{W}_{R}^{+} \cdot$ Antiparticles carry the Dirac charges $-D$ correspondingly. The Dirac charge of all SM particles vanishes. Note that the superpartners of left- and right-handed SM fermions carry opposite Dirac charge; this implies that terms mixing these fields will not conserve $D$. [Electroweak neutralinos and sleptons will be discussed in more detail in section 4 .] The Dirac charge $D$ conveniently classifies possible production processes and decay modes for the supersymmetric particles in the Dirac theory, as widely applied in the next sections. ${ }^{3}$

The cross sections for the processes $q q^{\prime} \rightarrow \tilde{q} \tilde{q}^{\prime}$ are characteristically different in the two limits [for simplicity, we again take equal masses for $\tilde{q}_{L}$ and $\left.\tilde{q}_{R}\right]$ :

$$
\begin{aligned}
& \text { Majorana }: \sigma\left[q q^{\prime} \rightarrow \tilde{q}_{L} \tilde{q}_{L}^{\prime}\right]=\sigma\left[q q^{\prime} \rightarrow \tilde{q}_{R} \tilde{q}_{R}^{\prime}\right]=\frac{2 \pi \alpha_{s}^{2}}{9} \frac{\beta m_{\tilde{g}_{1}}^{2}}{s m_{\tilde{g}_{1}}^{2}+\left(m_{\tilde{g}_{1}}^{2}-m_{\tilde{q}}^{2}\right)^{2}} \\
& \text { Dirac }: \sigma\left[q q^{\prime} \rightarrow \tilde{q}_{L} \tilde{q}_{L}^{\prime}\right]=\sigma\left[q q^{\prime} \rightarrow \tilde{q}_{R} \tilde{q}_{R}^{\prime}\right]=0 \\
& \text { Majorana = Dirac }: \sigma\left[q q^{\prime} \rightarrow \tilde{q}_{L} \tilde{q}_{R}^{\prime}\right]=\frac{2 \pi \alpha_{s}^{2}}{9 s^{2}}\left[\left(s+2\left(m_{\tilde{g}_{1}}^{2}-m_{\tilde{q}}^{2}\right)\right) L_{1}-2 \beta s\right],
\end{aligned}
$$

where $L_{1}$ has been defined in Eq.(3.8). The cross sections in the evolution from the Majorana limit to the Dirac limit are displayed in Fig. 3 at the parton level. While the $\tilde{q}_{L} \tilde{q}_{L}^{\prime}$ cross section moves monotonically to zero, the $\tilde{q}_{L} \tilde{q}_{R}^{\prime}$ cross section is only slightly modulated on the path $\mathcal{P}$ from the $\mathrm{N}=1$ Majorana limit to the Dirac limit. It should be noted that the Dirac cross sections are identical to the 2-Majorana cross sections owing to destructive interferences between the $\tilde{g}_{1}$ and $\tilde{g}_{2}$ exchange diagrams.

\footnotetext{
${ }^{3}$ We could equivalently define SM matter fermions to carry non-vanishing $D$, with $D\left[q_{L}\right]=-D\left[q_{R}\right]$, with sfermions having vanishing Dirac charge.
} 
Near-Dirac field: Generalizing the definitions Eqs. (3.21) and (3.22) for the Dirac field, the continuous approach of two nearly mass degenerate, nearly chirally coupled Majorana fields to the Dirac limit can be framed quantitatively. We define the fields

$$
\begin{aligned}
\tilde{g}_{\delta} & =\quad \sin \theta_{3} \tilde{g}_{1}-i \cos \theta_{3} \tilde{g}_{2} \equiv \cos \delta \tilde{g}_{D}+\sin \delta \tilde{g}_{D}^{c T}, \\
\tilde{g}_{\delta}^{c T} & =-\sin \theta_{3} \tilde{g}_{1}-i \cos \theta_{3} \tilde{g}_{2} \equiv \sin \delta \tilde{g}_{D}+\cos \delta \tilde{g}_{D}^{c T} .
\end{aligned}
$$

With $\cos / \sin \delta=\left(\cos \theta_{3} \pm \sin \theta_{3}\right) / \sqrt{2}$ they are identical to the standard Dirac fields for $\cos \theta_{3}=\sin \theta_{3}=1 / \sqrt{2}$, or $\delta=0$, but keep the approximate character of Dirac fields nearby $(0<|\delta| \ll 1)$. The contraction of the field with itself, $\sim \cos 2 \theta_{3}$, nearly vanishes for $\theta_{3} \sim \pi / 4$, while the contraction with the conjugate fields is unity. Thus the LL and RR transition amplitudes are proportional to $\cos 2 \theta_{3}$ and non-zero, while the LR transition remains 1 . The exchanges of the near-Dirac fields is equivalent to the exchanges of the two Majorana fields, generating transition amplitudes LL, $\mathrm{RR}=\cos ^{2} \theta_{3}-\sin ^{2} \theta_{3}=\cos 2 \theta_{3}$ and $\mathrm{LR}=\cos ^{2} \theta_{3}+\sin ^{2} \theta_{3}=1$. Taking the $\mathrm{N}=2$ gluino as an example in the limit $M_{3}^{\prime} \rightarrow 0$, the parameters describing the approach to the Dirac field are given by

$$
\begin{aligned}
& \cos \theta_{3}=\frac{1}{\sqrt{1+\left(1-M_{3}^{\prime} / m_{\tilde{g}_{1}}\right)^{2}}} \approx \frac{1}{\sqrt{2}}\left[1+\frac{1}{2} \frac{M_{3}^{\prime}}{m_{\tilde{g}_{1}}}\right] \\
& \sin \theta_{3}=\frac{1-M_{3}^{\prime} / m_{\tilde{g}_{1}}}{\sqrt{1+\left(1-M_{3}^{\prime} / m_{\tilde{g}_{1}}\right)^{2}}} \approx \frac{1}{\sqrt{2}}\left[1-\frac{1}{2} \frac{M_{3}^{\prime}}{m_{\tilde{g}_{1}}}\right],
\end{aligned}
$$

generating

$$
\cos \delta \approx 1 \quad \text { and } \quad \sin \delta \approx M_{3}^{\prime} / 2 m_{\tilde{g}_{1}} .
$$

In contrast to the wave functions, the two mass eigenvalues $m_{\tilde{g}_{1,2}}$ remain equal up to second order in $M_{3}^{\prime}$. As a result, exchanging the near-Dirac fields between L- and R-currents reproduces the cross sections calculated otherwise by the exchange of the almost degenerate Majorana fields.

\subsection{Summary of Characteristic Scattering Processes}

The entire ensemble of partonic cross sections for the N=1 Majorana theory has been calculated in Ref. [22], improving on the Born approximations [23] by including the radiative super-QCD corrections [for threshold resummations see [24]]. Electroweak tree-level contributions to the production of two (anti)squarks have been calculated in Ref. [25], while electroweak one-loop corrections to squark antisquark production have been derived in Ref. [26]. Since the number of reactions is approximately tripled when the theory is followed along the Majorana-Dirac path, we restrict the discussion to a set of characteristic examples. ${ }^{4}$ To highlight the characteristic differences between Majorana and Dirac theories, it is sufficient to work out the cross sections at the Born level.

\section{(a) Different-flavor quark scattering:}

These channels have been used in the previous sections to develop the differences between Majorana and Dirac theories. The results are presented in Eqs. (3.8), (3.27)-(3.29) and Fig. 3.

(b) Different-flavor quark-antiquark scattering:

The Feynman diagrams for $q \bar{q}^{\prime} \rightarrow \tilde{q}_{L} \tilde{q}_{L}^{\prime *}, \tilde{q}_{R} \tilde{q}_{R}^{*}, \tilde{q}_{L} \tilde{q}_{R}^{\prime *}$ are shown in Fig. 4 (a). In the Majorana and Dirac limits, the

4 The complete set of cross sections is available at http://www.pitt.edu/ a afreitas/formulas.pdf. 


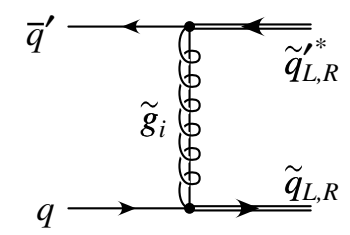

(a)

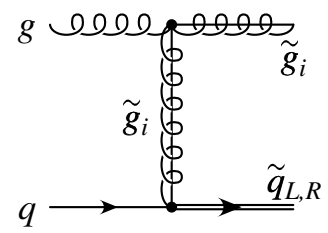

(b)

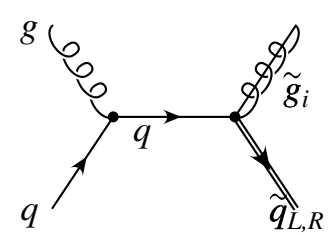

(c)

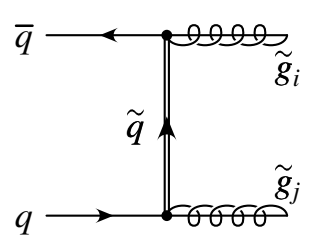

(d)

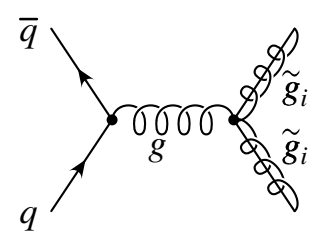

(e)

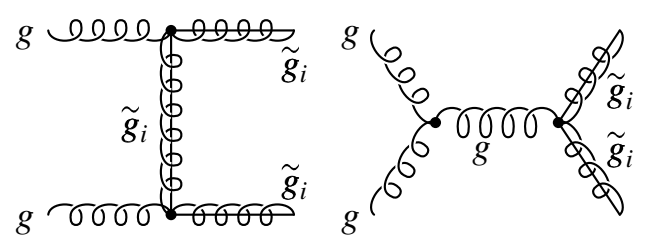

(f)

(g)

FIG. 4: Feynman diagrams for different-flavor squark production in quark annihilation (a), squark-gluino production (b,c), gluino production in quark annihilation $(\mathrm{d}, \mathrm{e})$ and gluino production in gluon fusion $(\mathrm{f}, \mathrm{g})$. [The indices $i, j$ count the two gluinos in the $\mathrm{N}=2$ hybrid model, and should be ignored for the $\mathrm{N}=1 \mathrm{MSSM}$.]

partonic cross sections read

$$
\begin{aligned}
\text { Majorana }=\text { Dirac }: \sigma\left[q \bar{q}^{\prime} \rightarrow \tilde{q}_{L} \tilde{q}_{L}^{\prime *}\right] & =\sigma\left[q \bar{q}^{\prime} \rightarrow \tilde{q}_{R} \tilde{q}_{R}^{\prime *}\right]=\frac{2 \pi \alpha_{s}^{2}}{9 s^{2}}\left[\left(s+2\left(m_{\tilde{g}_{1}}^{2}-m_{\tilde{q}}^{2}\right)\right) L_{1}-2 \beta s\right] \\
\text { Majorana }: & \sigma\left[q \bar{q}^{\prime} \rightarrow \tilde{q}_{L} \tilde{q}_{R}^{\prime *}\right]=\frac{2 \pi \alpha_{s}^{2}}{9} \frac{\beta m_{\tilde{g}_{1}}^{2}}{s m_{\tilde{g}_{1}}^{2}+\left(m_{\tilde{g}_{1}}^{2}-m_{\tilde{q}}^{2}\right)^{2}} \\
\text { Dirac }: \sigma\left[q \bar{q}^{\prime} \rightarrow \tilde{q}_{L} \tilde{q}_{R}^{\prime *}\right] & =0
\end{aligned}
$$

As before, $\beta=\left(1-4 m_{\tilde{q}}^{2} / s\right)^{1 / 2}$ is the velocity of the produced squarks. Numerical results for the cross sections along the path $-1 \rightarrow y \rightarrow 0$ are displayed in Fig. 5 (a).

For equal-flavor quark-antiquark scattering the additional gluino $s$-channel exchange must be added to the $t$-channel exchange diagrams.

\section{(c) Squark-gluino production:}

The Feynman diagrams for the super/hyper-Compton processes $g q \rightarrow \tilde{q} \tilde{g}_{1}, \tilde{q} \tilde{g}_{2}$ are given in Fig. 4 (b,c). As before, we give formulas for the cross sections in the two limiting cases:

$$
\begin{aligned}
\text { Majorana }=\text { Dirac }: \begin{aligned}
\sigma\left[g q \rightarrow \tilde{q}_{L, R} \tilde{g}\right]= & \sigma\left[g q \rightarrow \tilde{q}_{L} \tilde{g}_{D}\right]=\sigma\left[g q \rightarrow \tilde{q}_{R} \tilde{g}_{D}^{c}\right] \\
= & \frac{\pi \alpha_{s}^{2}}{18 s^{3}}\left[2\left(4 s-4 m_{\tilde{g}_{1}}^{2}-5 m_{\tilde{q}}^{2}\right)\left(m_{\tilde{g}_{1}}^{2}-m_{\tilde{q}}^{2}\right) L_{1}^{\prime}\right. \\
& +9\left(s\left(s+2 m_{\tilde{g}_{1}}^{2}\right)+2 m_{\tilde{q}}^{2}\left(m_{\tilde{q}}^{2}-m_{\tilde{g}_{1}}^{2}-s\right)\right) L_{1} \\
& \left.-\beta s\left(7 s+32\left(m_{\tilde{g}_{1}}^{2}-m_{\tilde{q}}^{2}\right)\right)\right]
\end{aligned} \\
\text { Dirac : } \sigma\left[g q \rightarrow \tilde{q}_{L} \tilde{g}_{D}^{c}\right]=\sigma\left[g q \rightarrow \tilde{q}_{R} \tilde{g}_{D}\right]=0,
\end{aligned}
$$

with

$$
L_{1}=\log \frac{(1+\beta)+2\left(m_{\tilde{g}_{1}}^{2}-2 m_{\tilde{q}}^{2}\right) / s}{(1-\beta)+2\left(m_{\tilde{g}_{1}}^{2}-2 m_{\tilde{q}}^{2}\right) / s} \quad L_{1}^{\prime}=\log \frac{(1+\beta)-2\left(m_{\tilde{g}_{1}}^{2}-2 m_{\tilde{q}}^{2}\right) / s}{(1-\beta)-2\left(m_{\tilde{g}_{1}}^{2}-2 m_{\tilde{q}}^{2}\right) / s}
$$

Here $\beta=\left(\left[s-\left(m_{\tilde{q}}+m_{\tilde{g}_{1}}\right)^{2}\right]\left[s-\left(m_{\tilde{q}}-m_{\tilde{g}_{1}}\right)^{2}\right]\right)^{1 / 2} / s$ denotes the momenta of the final-state squarks and gluinos in units of half the total c.m. parton energy, i.e. the velocity for equal-mass particles. Fig. 5 (b) shows the cross sections for the two Majorana mass eigenstates along the interpolated path between the two limits. As can be seen in the 
(a)
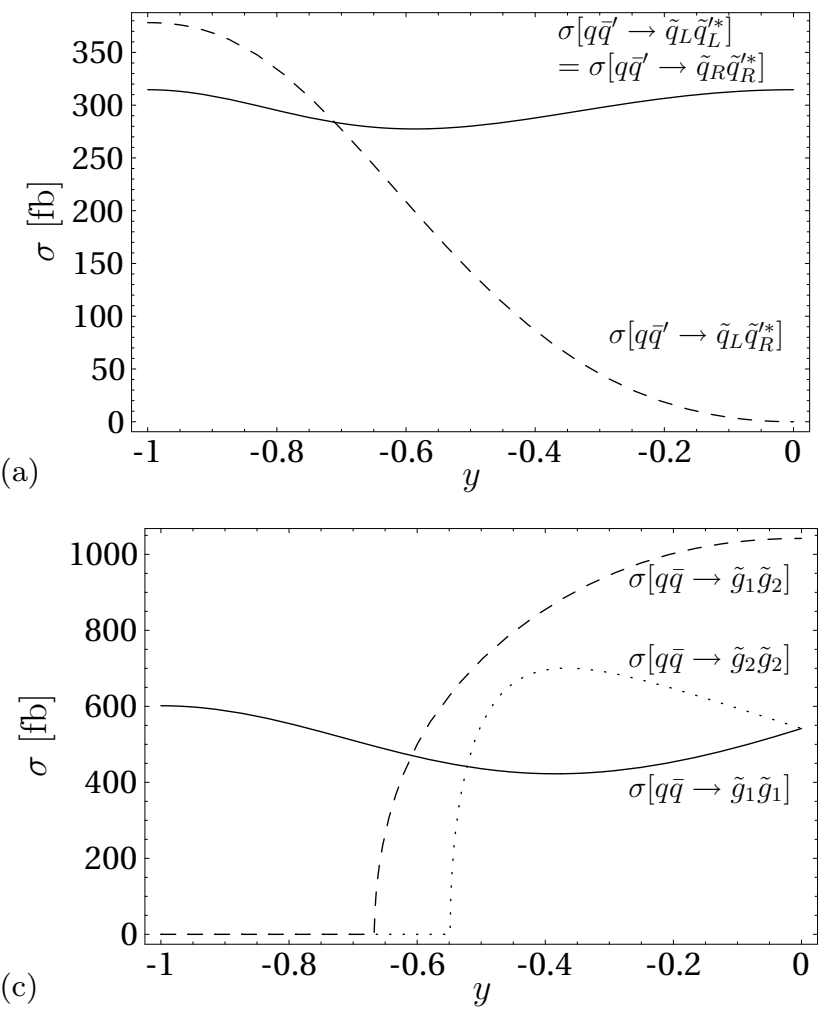
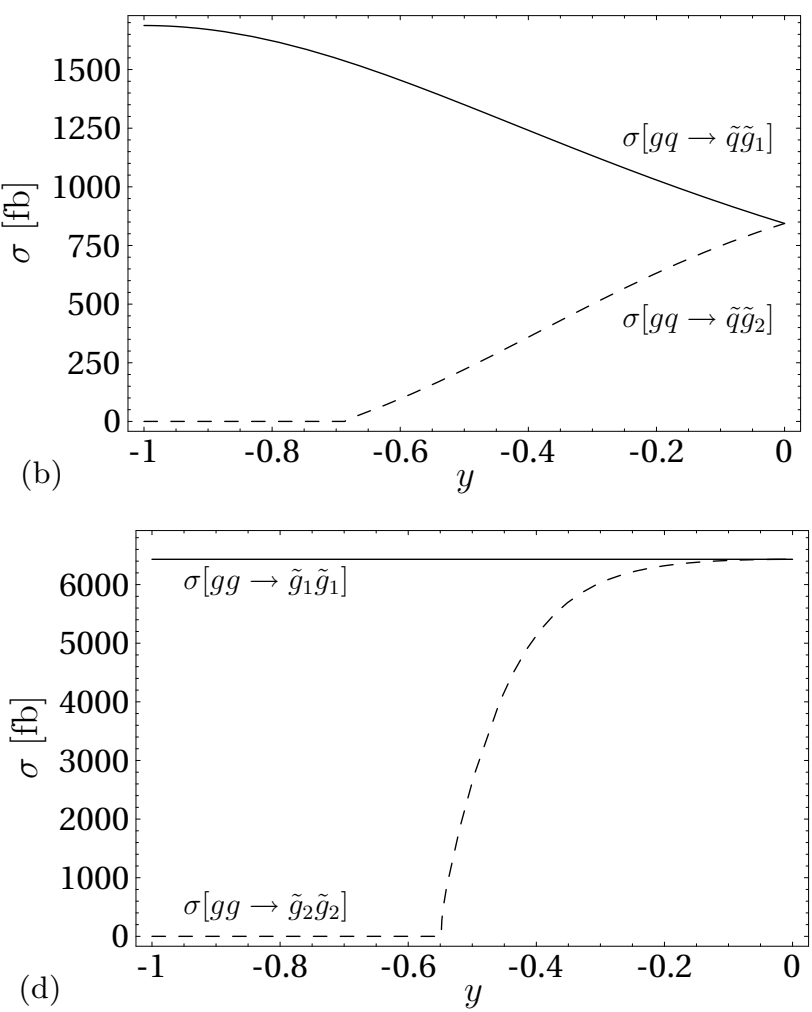

FIG. 5: Partonic cross sections for different-flavor squark production in quark annihilation (a), squark-gluino production (b) and gluino production (c,d). The cross sections are given as functions of the Dirac/Majorana control parameter $y$. Soft breaking parameters are as in Fig. 3.

figure, the second gluino $\tilde{g}_{2}$ can only be produced if, for the fixed parton c.m. energy, it becomes light enough so that the kinematical threshold is crossed. Approaching the Dirac limit $y \rightarrow 0$, the cross sections for $\tilde{q} \tilde{g}_{1}$ and $\tilde{q} \tilde{g}_{2}$ production become equal. Note that the total $\tilde{q} \tilde{g}$ production cross section is the same in the Dirac limit as in the original MSSM.

\section{(d) Gluino pairs:}

Gluino pairs can be produced through quark annihilation, $q \bar{q} \rightarrow \tilde{g}_{1} \tilde{g}_{1}, \tilde{g}_{1} \tilde{g}_{2}, \tilde{g}_{2} \tilde{g}_{2}$ (see Fig. 4 (d,e)), or through gluon fusion $g g \rightarrow \tilde{g}_{1} \tilde{g}_{1}, \tilde{g}_{2} \tilde{g}_{2}$ (see Fig. 4 (f,g)). The production of gluino pairs in gluon-gluon collisions is based solely on QCD gauge interactions. By conservation of the color current, mixed $\tilde{g}_{1}, \tilde{g}_{2}$ gluino pair production is therefore not possible. The cross sections are given by

$$
\begin{aligned}
& \text { Majorana }: \quad \sigma[q \bar{q} \rightarrow \tilde{g} \tilde{g}]=\frac{4 \pi \alpha_{s}^{2}}{27 s^{3}}\left[-2 \frac{8 s^{2} m_{\tilde{q}}^{2}+s\left(7 m_{\tilde{g}_{1}}^{4}-32 m_{\tilde{g}_{1}}^{2} m_{\tilde{q}}^{2}+25 m_{\tilde{q}}^{4}\right)-18\left(m_{\tilde{g}_{1}}^{2}-m_{\tilde{q}}^{2}\right)^{3}}{s-2 m_{\tilde{g}_{1}}^{2}+2 m_{\tilde{q}}^{2}} L_{1}^{\prime}\right. \\
& \left.+\beta\left(13 s^{2}-6 s\left(m_{\tilde{g}_{1}}^{2}-3 m_{\tilde{q}}^{2}\right)-\frac{8 s^{3} m_{\tilde{q}}^{2}}{s m_{\tilde{q}}^{2}+\left(m_{\tilde{g}_{1}}^{2}-m_{\tilde{q}}^{2}\right)^{2}}\right)\right] \\
& \left.+\beta\left(19 s^{2}+6 s\left(m_{\tilde{g}_{1}}^{2}+3 m_{\tilde{q}}^{2}\right)-\frac{8 s^{3} m_{\tilde{q}}^{2}}{s m_{\tilde{q}}^{2}+\left(m_{\tilde{g}_{1}}^{2}-m_{\tilde{q}}^{2}\right)^{2}}\right)\right] \\
& \sigma\left[q \bar{q} \rightarrow \tilde{g}_{D} \tilde{g}_{D}\right]=\sigma\left[q \bar{q} \rightarrow \tilde{g}_{D}^{c} \tilde{g}_{D}^{c}\right]=0
\end{aligned}
$$


and

$$
\begin{aligned}
\text { Majorana }: \sigma[g g \rightarrow \tilde{g} \tilde{g}] & =\frac{3 \pi \alpha_{s}^{2}}{4 s^{3}}\left[3\left(s^{2}+4 s m_{\tilde{g}_{1}}^{2}-4 m_{\tilde{g}_{1}}^{4}\right) \log \frac{1+\beta}{1-\beta}-\beta s\left(4 s+17 m_{\tilde{g}_{1}}^{2}\right)\right] \\
\text { Dirac }: \sigma\left[g g \rightarrow \tilde{g}_{D} \tilde{g}_{D}^{c}\right] & =\frac{3 \pi \alpha_{s}^{2}}{2 s^{3}}\left[3\left(s^{2}+4 s m_{\tilde{g}_{1}}^{2}-4 m_{\tilde{g}_{1}}^{4}\right) \log \frac{1+\beta}{1-\beta}-\beta s\left(4 s+17 m_{\tilde{g}_{1}}^{2}\right)\right] \\
\sigma\left[g g \rightarrow \tilde{g}_{D} \tilde{g}_{D}\right] & =\sigma\left[g g \rightarrow \tilde{g}_{D}^{c} \tilde{g}_{D}^{c}\right]=0,
\end{aligned}
$$

in the same notation as before, with $L_{1}^{\prime}$ defined in $(3.39)$ but $\beta=\left(1-4 m_{\tilde{g}_{1}}^{2} / s\right)^{1 / 2}$. As the nature of the gluino is changed smoothly from Majorana to Dirac along the path $\mathcal{P}$, several thresholds are crossed for fixed parton c.m. energy, see Fig. 5 (c,d), whenever the second Majorana particle becomes light enough to allow $\tilde{g}_{1} \tilde{g}_{2}$ and $\tilde{g}_{2} \tilde{g}_{2}$ pair production, respectively.

Again, the identity of the 2-Majorana with the Dirac theory can be re-examined by verifying the equality of the cross sections,

$$
\begin{aligned}
\sigma\left[q \bar{q} \rightarrow \tilde{g}_{D} \tilde{g}_{D}^{c}\right] & =\sum_{k, l=1}^{2} \sigma\left[q \bar{q} \rightarrow \tilde{g}_{k} \tilde{g}_{l}\right], \\
\sigma\left[g g \rightarrow \tilde{g}_{D} \tilde{g}_{D}^{c}\right] & =\sum_{k}^{2} \sigma\left[g g \rightarrow \tilde{g}_{k} \tilde{g}_{k}\right],
\end{aligned}
$$

in a meticulous accounting of interference effects in double-gluino production. In the Dirac limit, the total production cross section for $g g \rightarrow$ gluinos is therefore twice as large as in the MSSM.

The hadron cross sections will be discussed for the LHC in the final section, including crucial tests for discriminating the MSSM Majorana theory from a Dirac theory experimentally.

\subsection{Gluino Decays}

If squarks are heavier than gluinos the dominant channels are decays to gluinos. Otherwise squarks decay into electroweak chargino and neutralino channels. Gluinos in turn always decay to pairs of quarks and squarks, either real or virtual. The partial widths of all these strong [27] and electroweak modes [28] are known in next-to-leading order in $\mathrm{N}=1$ supersymmetry. In this subsection only strong decay channels will be discussed, while electroweak decays are postponed to the next section.

(a) $m_{\tilde{q}}>m_{\tilde{g}}$ : The partial widths for squark decays to Majorana and Dirac gluinos,

$$
\begin{aligned}
\Gamma\left[\tilde{q}_{L} \rightarrow q \tilde{g}\right]=\Gamma\left[\tilde{q}_{R} \rightarrow q \tilde{g}\right]=\Gamma\left[\tilde{q}_{L} \rightarrow q \tilde{g}_{D}^{c}\right] & =\Gamma\left[\tilde{q}_{R} \rightarrow q \tilde{g}_{D}\right]=\frac{2 \alpha_{s}}{3} \frac{\left(m_{\tilde{q}}^{2}-m_{\tilde{g}}^{2}\right)^{2}}{m_{\tilde{q}}^{3}}, \\
\Gamma\left[\tilde{q}_{R} \rightarrow q \tilde{g}_{D}^{c}\right] & =\Gamma\left[\tilde{q}_{L} \rightarrow q \tilde{g}_{D}\right]=0
\end{aligned}
$$

[and correspondingly for the charge-conjugate states] are the same for equal masses and couplings. This applies for the two endpoints of the path $\mathcal{P}$, the standard $\mathrm{N}=1$ Majorana limit and the Dirac limit. Even though the decay mechanism is strong, the $\mathrm{P}$-wave decay width is suppressed nevertheless when the squark/gluino mass difference becomes small.

(b) $m_{\tilde{g}}>m_{\tilde{q}}$ : A similar relation applies for Majorana and Dirac gluino decays into quarks and squarks [and charge-conjugate states]:

$$
\begin{aligned}
\Gamma\left[\tilde{g} \rightarrow q \tilde{q}_{L}^{*}\right]=\Gamma\left[\tilde{g} \rightarrow q \tilde{q}_{R}^{*}\right]=\Gamma\left[\tilde{g}_{D} \rightarrow q \tilde{q}_{L}^{*}\right] & =\Gamma\left[\tilde{g}_{D}^{c} \rightarrow q \tilde{q}_{R}^{*}\right]=\frac{\alpha_{s}}{8} \frac{\left(m_{\tilde{g}}^{2}-m_{\tilde{q}}^{2}\right)^{2}}{m_{\tilde{g}}^{3}} \\
\Gamma\left[\tilde{g}_{D} \rightarrow q \tilde{q}_{R}^{*}\right] & =\Gamma\left[\tilde{g}_{D}^{c} \rightarrow q \tilde{q}_{L}^{*}\right]=0
\end{aligned}
$$


Non-isotropic angular distributions follow the familiar $\cos \theta$ distribution for spin $1 / 2 \rightarrow \operatorname{spin} 1 / 2+$ spin 0 decays.

Squarks decay either to Dirac particles or to Dirac antiparticles. These modes can be distinguished in the subsequent Dirac decays.

The Majorana or Dirac character of the gluinos can be demonstrated nicely in the charge assignments of squarks in the decays of gluino pairs:

$$
\begin{aligned}
\text { Majorana: } & \tilde{g} \rightarrow q_{L} \tilde{q}_{L}^{*} \oplus \overline{q_{L}} \tilde{q}_{L} \text { and } q_{R} \tilde{q}_{R}^{*} \oplus \overline{q_{R}} \tilde{q}_{R} \\
\text { Dirac: } & \tilde{g}_{D} \rightarrow q_{L} \tilde{q}_{L}^{*} \text { and } \overline{q_{R}} \tilde{q}_{R} \\
& \tilde{g}_{D}^{c} \rightarrow \overline{q_{L}} \tilde{q}_{L} \text { and } q_{R} \tilde{q}_{R}^{*},
\end{aligned}
$$

where $\oplus$ connects final states that are produced at equal rates.

For the first two generations, mixing between L- and R-squarks is expected to be negligible. In this case, the chiralities of the squarks can be distinguished clearly by their decay modes. For instance, if the lightest neutralino is mainly bino and the next-to-lightest neutralino is dominantly wino, the L-squarks have sizable branching fractions into decay cascades leading to additional leptons, $\tilde{q}_{L} \rightarrow q \tilde{\chi}_{2}^{0} \rightarrow q l^{+} l^{-} \tilde{\chi}_{1}^{0}$ or $\tilde{q}_{L} \rightarrow q \tilde{\chi}_{1}^{ \pm} \rightarrow q l^{ \pm} \nu_{l} \tilde{\chi}_{1}^{0}, l=e, \mu, \tau$. On the other hand, R-squarks would almost always decay directly to the lightest neutralino, $\tilde{q}_{R} \rightarrow q \tilde{\chi}_{1}^{0}$. Furthermore, the decay chain $\tilde{q}_{L} \rightarrow q \tilde{\chi}_{1}^{ \pm} \rightarrow q l^{ \pm} \nu_{l} \tilde{\chi}_{1}^{0}$ allows to determine the charge of the $\tilde{q}_{L}$ experimentally.

Production of Majorana gluino pairs leads to equal amounts of same-sign and opposite-sign L-squarks, while Dirac gluino pairs generate only the ordinary opposite-sign combination:

$$
\begin{aligned}
\text { Majorana : } \quad p p \rightarrow \tilde{g} \tilde{g} & \rightarrow q q \tilde{q}_{L}^{*} \tilde{q}_{L}^{*} \oplus q \bar{q} \tilde{q}_{L} \tilde{q}_{L}^{*} \oplus \bar{q} q \tilde{q}_{L}^{*} \tilde{q}_{L} \oplus \bar{q} \bar{q} \tilde{q}_{L} \tilde{q}_{L} \\
\text { Dirac }: \quad p p & \rightarrow \tilde{g}_{D} \tilde{g}_{D}=0 \\
p p & \rightarrow \tilde{g}_{D} \tilde{g}_{D}^{c} \rightarrow q \bar{q} \tilde{q}_{L} \tilde{q}_{L}^{*},
\end{aligned}
$$

and correspondingly for R-squarks and mixed L/R final states. In section 5 the LHC phenomenology of this process will be discussed in more detail.

For gluino decays into tops and stops the situation is more complex due to potentially sizable stop mixing. Nevertheless, unless the stop mixing is maximal, i.e. $\theta_{\tilde{t}}=\pi / 4$, Dirac gluinos will lead to an asymmetry in the stop charge assignment as a result of mass difference between the two stop mass eigenstates:

$$
\begin{aligned}
\text { Majorana: } \quad p p \rightarrow \tilde{g} \tilde{g} & \rightarrow t t \tilde{t}^{*} \tilde{t}^{*} \oplus t \bar{t} \tilde{t} \tilde{t}^{*} \oplus \bar{t} t \tilde{t}^{*} \tilde{t} \oplus \bar{t} \bar{t} \tilde{t} \tilde{t} \\
\text { Dirac }: \quad p p \rightarrow \tilde{g}_{D} \tilde{g}_{D} & =0 \\
p p & \rightarrow \tilde{g}_{D} \tilde{g}_{D}^{c} \rightarrow \alpha_{D}\left(t_{L} t_{R} \tilde{t}^{*} \tilde{t}^{*} \oplus \overline{t_{L}} \overline{t_{R}} \tilde{t} \tilde{t}\right) \text { and } \beta_{D}\left(t_{L} \overline{t_{L}} \tilde{t} \tilde{t}^{*} \oplus \overline{t_{R}} t_{R} \tilde{t}^{*} \tilde{t}\right) .
\end{aligned}
$$

The gluinos will decay with a larger branching fraction into the lighter of the two stop states. For Majorana pairs this leads to universal charge assignments independent of stop mixing. On the other hand, a Dirac gluino $\tilde{g}_{D}$ (antigluino $\tilde{g}_{D}^{c}$ ) decays more often into a stop (antistop) if the lighter stop state is mostly R-chiral. If the lighter stop is mostly L-chiral, the opposite decay patterns dominate. Either way one obtains

$$
\alpha_{D}<\beta_{D}
$$

leading to more opposite-sign top pairs than same-sign top pairs in the final state. In addition, the Majorana gluinos $\tilde{g}$ decay to top and antitop quarks of both chiralities L,R with equal probability while the Dirac gluino pairs $\tilde{g}_{D} \tilde{g}_{D}^{c}$ decay to quarks [or antiquarks] which carry different L and R chiralities as indicated in Eq.(3.54), giving rise to different decay distributions. 
In the Dirac theory the "Majorana-like" decay pattern $\alpha_{D}=\beta_{D}$ can only be realized for maximal stop mixing. Using leptonic decay modes of the top quarks to identify their charge, multi-top final states therefore offer a powerful testing ground for distinguishing Majorana from Dirac gluinos.

\section{THE ELECTROWEAK SECTOR}

If for $\mathrm{N}=1$ the supersymmetry breaking scale is much larger than the electroweak mass scale $v$, the neutralino sector includes two Majorana gauginos associated with the hypercharge $\mathrm{U}(1)$ and the isospin $\mathrm{SU}(2)$ gauge groups, and two nearly mass degenerate Majorana higgsinos. Thus, in the limit $v / \mu \rightarrow 0$ the system consists of two Majorana gauginos and one Dirac higgsino. Extending the $\mathrm{N}=1$ supersymmetry to the $\mathrm{N}=2$ supersymmetry, the two gaugino degrees of freedom are doubled and, in parallel to the gluino sector, the two $\mathrm{U}(1)$ and $\mathrm{SU}(2)$ related gaugino fields may transform from Majorana to Dirac fields. ${ }^{5}$

\section{1. $\mathrm{N}=1 / \mathrm{N}=2$ Neutralino and Chargino Masses and Spinor Wave Functions}

In the limit of asymptotically high $\mathrm{N}=2$ supersymmetry scales, the neutralino mass matrix (2.10) disintegrates into three weakly coupled $2 \times 2$ sub-matrices associated with the gauginos of the gauge groups $\mathrm{U}(1)$ and $\mathrm{SU}(2)$, and the higgsino sector. If the new gaugino mass parameters $M_{1,2}^{\prime}$ are infinitely large, the system is reduced to the familiar $\mathrm{N}=1$ MSSM. On the other hand, if the on-diagonal elements of the two $2 \times 2$ gaugino sub-matrices vanish and the sub-matrices are reduced to equal off-diagonal elements, the two Majorana fields of each group can be joined to a Dirac field. In the limit $v \rightarrow 0$ the mechanisms operate strictly parallel to the gluino sector.

Since the N=1 Majorana limit for neutralinos has been worked out in all of its facets in the past, we will here restrict ourselves solely to the discussion of the Dirac/near-Dirac limit. The original current fields in Cartesian coordinates are denoted by

$$
\tilde{\chi}_{\text {curr }}=\left\{\tilde{B}^{\prime}, \tilde{B}, \tilde{W}^{\prime 3}, \tilde{W}^{3}, \tilde{H}_{d}, \tilde{H}_{u}\right\}^{T}
$$

the mass eigenfields, for $v \rightarrow 0$, are maximally mixed superpositions of the current eigenfields:

$$
\tilde{\chi}_{\text {mass }}=\left\{\tilde{B}_{1}, \tilde{B}_{2}, \tilde{W}_{1}^{3}, \tilde{W}_{2}^{3}, \tilde{H}_{1}, \tilde{H}_{2}\right\}^{T},
$$

where, for real and non-negative $M_{1}^{D}, M_{2}^{D}$ and $\mu$, the six mass eigenstates are written in terms of the current fields as

$$
\begin{aligned}
& \tilde{B}_{1,2}=\{i\}\left[\left(\tilde{B}_{L}^{\prime} \pm \tilde{B}_{R}^{\prime}\right) \pm\left(\tilde{B}_{L} \pm \tilde{B}_{R}\right)\right] / \sqrt{2} \\
& \tilde{W}_{1,2}=\{i\}\left[\left(\tilde{W}_{L}^{\prime} \pm \tilde{W}_{R}^{\prime}\right) \pm\left(\tilde{W}_{L} \pm \tilde{W}_{R}\right)\right] / \sqrt{2} \\
& \tilde{H}_{2,1}=\{i\}\left[\left(\tilde{H}_{u L} \pm \tilde{H}_{u R}\right) \mp\left(\tilde{H}_{d L} \pm \tilde{H}_{d R}\right)\right] / \sqrt{2}
\end{aligned}
$$

with mass eigenvalues $m_{\tilde{B}_{1,2}}=M_{1}^{D}, m_{\tilde{W}_{1,2}^{3}}=M_{2}^{D}$ and $m_{\tilde{H}_{1,2}}=|\mu|$, respectively. [The coefficient $\{i\}$ is associated with the second entry in each row.]

The neutral Majorana fields can be joined pairwise to form three Dirac fields in the $v=0$ limit:

$$
\tilde{\chi}_{D}^{0}=\left\{\tilde{B}_{D}, \tilde{W}_{D}^{3}, \tilde{H}_{D}\right\}
$$

\footnotetext{
5 The discussion of the electroweak sector is restricted, almost exclusively, to those points which affect the phenomenology of squark/gluino decays; the only exception will be selectron pair production for polarized beams.
} 
where the Dirac fields are expressed in terms of the mass eigenfields as

$$
\begin{aligned}
\tilde{W}_{D}^{3} & =\left(\tilde{W}_{1}^{3}-i \tilde{W}_{2}^{3}\right) / \sqrt{2} \quad \text { and } \quad \tilde{W}^{3} \Rightarrow \tilde{B} \\
\tilde{H}_{D}^{0} & =\left(\tilde{H}_{1}-i \tilde{H}_{2}\right) / \sqrt{2}
\end{aligned}
$$

while the corresponding charge-conjugated fields read:

$$
\begin{aligned}
& \tilde{W}_{D}^{3 c}=-\left(\tilde{W}_{1}^{3}+i \tilde{W}_{2}^{3}\right) / \sqrt{2} \quad \text { and } \quad \tilde{W}^{3 c} \Rightarrow \tilde{B}^{c}, \\
& \tilde{H}_{D}^{0 c}=+\left(\tilde{H}_{1}+i \tilde{H}_{2}\right) / \sqrt{2} .
\end{aligned}
$$

The charged Dirac fields, in parallel to the neutral fields but in circular notation, are given by

$$
\begin{aligned}
& \tilde{W}_{1,2}^{ \pm}=\{i\}\left[\left(\tilde{W}_{L}^{\prime \pm} \pm \tilde{W}_{R}^{\prime \pm}\right) \pm\left(\tilde{W}_{L}^{ \pm} \pm \tilde{W}_{R}^{ \pm}\right)\right] / \sqrt{2} \\
& \tilde{H}^{ \pm}=\tilde{H}_{u L / R}^{ \pm}+\tilde{H}_{d R / L}^{ \pm}
\end{aligned}
$$

These fields are mutually conjugate to each other. The \pm fields can be rotated to three new charged Dirac fields:

$$
\begin{aligned}
& \tilde{\chi}_{D 1}^{ \pm}=\left(\tilde{W}_{1}^{ \pm} \pm i \tilde{W}_{2}^{ \pm}\right) / \sqrt{2}, \\
& \tilde{\chi}_{D 2}^{ \pm}=\left(\tilde{W}_{1}^{ \pm} \mp i \tilde{W}_{2}^{ \pm}\right) / \sqrt{2}, \\
& \tilde{\chi}_{D 3}^{ \pm}=\tilde{H}^{ \pm},
\end{aligned}
$$

generating, in association of the charged gaugino and higgsino fields, an ensemble of three chargino fields. Again the \pm components are related by $C$-conjugation.

In the limit of small but non-zero $v$, all the fields are weakly mixed after electroweak symmetry breaking, i.e. the original mass eigenfields defined in Eqs. (4.5) and (4.8) receive small admixtures. The final neutralino mass eigenfields may be written, up to terms linear in $v / M_{\mathrm{SUSY}}$ :

$$
\tilde{\chi}_{\mathrm{phys}} \approx\left(\begin{array}{cc}
\mathbb{1}_{4 \times 4} & -\Omega_{D} \\
\Omega_{D}^{\dagger} & \mathbb{1}_{2 \times 2}
\end{array}\right) \tilde{\chi}_{\mathrm{mass}}
$$

with the $4 \times 2$ matrix $\Omega_{D}$ accounting for the admixture between gauginos and higgsinos,

$$
\Omega_{D}=m_{Z}\left(\begin{array}{rr}
i s_{W} s_{\beta} / \mu_{1+} & s_{W} c_{\beta} / \mu_{1-} \\
-s_{W} c_{\beta} / \mu_{1-} & -i s_{W} s_{\beta} / \mu_{1+} \\
-i c_{W} s_{\beta} / \mu_{2+} & -c_{W} c_{\beta} / \mu_{2-} \\
c_{W} c_{\beta} / \mu_{2-} & i c_{W} s_{\beta} / \mu_{2+}
\end{array}\right)
$$

with $\mu_{1+}=\mu+M_{1}^{D}$ etc. For the chargino states, one finds similarly:

$$
\tilde{\chi}_{\mathrm{phys}}^{ \pm} \approx\left(\begin{array}{cc}
\mathbb{1}_{2 \times 2} & -\Omega_{ \pm} \\
\Omega_{ \pm}^{\dagger} & 1
\end{array}\right) \tilde{\chi}_{\text {mass }}^{ \pm}
$$

with the $2 \times 1$ matrix $\Omega_{ \pm}$taking into account the small mixing between gauginos and higgsinos,

$$
\Omega_{ \pm}=m_{W}\left(\begin{array}{r}
c_{\beta} / \mu_{2-} \mp s_{\beta} / \mu_{2+} \\
-i s_{\beta} / \mu_{2+} \mp i c_{\beta} / \mu_{2-}
\end{array}\right) .
$$

Up to linear accuracy in $v / M_{\mathrm{SUSY}}$ the neutralino and chargino mass eigenvalues are unaltered.

The Dirac charge has been introduced for convenient book-keeping of allowed and forbidden reactions in the $\mathrm{N}=2$ hybrid theory. Of course, the charginos form Dirac fields even in the MSSM. However, for non-zero masses one cannot define a conserved Dirac charge in this more restricted theory. The gauge-strength Yukawa-type couplings of the 
charginos to a sfermion and an outgoing left-handed matter fermion involve both the $\mathrm{L}$ and $\mathrm{R}$ components of the

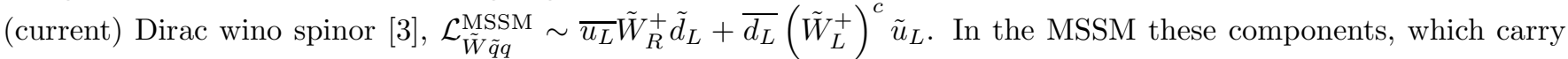
opposite $D$-charge, are coupled by the mass $M_{2}$. In contrast, in the $\mathrm{N}=1 / \mathrm{N}=2$ hybrid theory a conserved Dirac charge (3.26) can be defined for $v \rightarrow 0$, since the $\mathrm{L}$ and $\mathrm{R}$ components of the original $\mathrm{N}=1$ wino $\tilde{W}$ belong to different Dirac fields in this limit. Since no second "partner" field has been introduced in the higgsino sector, their couplings to fermions and sfermions, which are determined by the standard Yukawa interactions, will not conserve $D$ either. The transition to mixed gaugino/higgsino states will be discussed in a sequel to this report.

The formalism can now be applied to compare signatures distinguishing the original $\mathrm{N}=1$ Majorana theory from a Dirac theory in the electroweak sector as formulated explicitly in the hybrid model.

\subsection{Electroweak Squark Cascade Decays in Majorana and Dirac Scenarios}

The generic structures of sfermion decays to neutralinos/charginos and of neutralino/chargino decays to sfermion plus fermion pairs are similar to those of squark and gluino decays in super- and hyper-QCD. The complexity increases due to the mixing between gauginos and higgsinos and between left- and right-handed sfermions originating from electroweak symmetry breaking. However, for the first and second generation (s)fermions with small Yukawa couplings the contamination is negligible.

A rich ensemble of observables for measuring the properties of supersymmetric particles at the LHC is provided by cascade decays involving neutralinos. In particular, the squark cascades with intermediate neutralinos and sleptons have served to study experimental prospects of measuring masses and spins. In addition, the Majorana or Dirac nature of the neutralinos can be determined by measuring the distributions of the charged leptons in the final state.

In the following discussion we assume that only $\mathrm{SU}(2)$ singlet sleptons $\tilde{l}_{R}$ are accessible in the decay of the relevant neutralino $\tilde{\chi}_{2}^{0}$. Ignoring lepton mass effects, the charged "near" lepton produced together with the slepton is then either a left-handed $l^{+}$or a right-handed $l^{-}$. [We will see in a moment that only one of these possibilities is allowed in the Dirac theory.] Neutralinos produced in $\tilde{q}_{L}$ decays are produced in association with a left-handed quark, i.e. they are predominantly left-handed. Angular momentum conservation then implies that a near $l^{-}\left[l^{+}\right]$preferentially goes opposite [parallel] to the neutralino flight direction. In the rest frame of the decaying $\tilde{q}_{L}$ a near $l^{-}$will thus tend to be softer, and closer to the quark in phase space, than a near $l^{+}$. These correlations are reflected in the invariant $q l$ mass distributions $[29,30]$. The same argument implies that the slepton, and hence the "far" lepton that results from its decay, will be harder [softer] if it has positive [negative] charge.

In Majorana theories the neutralino $\tilde{\chi}_{2}^{0}$ can decay into sleptons $\tilde{l}_{R}$ of both positive and negative charge:

$$
\tilde{q}_{L} \rightarrow q \tilde{\chi}_{2}^{0} \rightarrow q l_{n}^{\mp} \tilde{l}_{R}^{ \pm} \rightarrow q l_{n}^{\mp} l_{f}^{ \pm} \tilde{\chi}_{1}^{0} .
$$

The near $(n)$ leptons and the far $(f)$ leptons, produced directly in the $\tilde{\chi}_{2}^{0}$ decays and in the subsequent $\tilde{l}_{R}$ decays respectively, both can have either negative or positive charges, albeit with different energy distributions as a result of the neutralino polarization discussed above.

By contrast, the transition from Majorana to Dirac particles leads to a simpler situation. In the Dirac theory, evaluating the generic fermion-sfermion-neutralino Lagrangian, restricted to gauginos for the first two generations, results in

$$
\mathcal{L}^{f \tilde{f} \tilde{\chi}}=g_{L}\left(\overline{f_{L}} \tilde{\chi}_{D}^{0} \tilde{f}_{L}+\overline{\tilde{\chi}_{D}^{0}} f_{L} \tilde{f}_{L}^{*}\right)+g_{R}\left(\overline{f_{R}} \tilde{\chi}_{D}^{c 0} \tilde{f}_{R}+\overline{\tilde{\chi}_{D}^{c 0}} f_{R} \tilde{f}_{R}^{*}\right)
$$

[The L- and R-couplings $g_{L}, g_{R}$ are defined in terms of the neutralino mixing matrix and the fermion isospin and hyper-charges, as frequently noted in the literature; recall that $D\left[\tilde{\chi}_{D}^{0}\right]=-D\left[\tilde{\chi}_{D}^{c 0}\right]=+1$.] A fixed sequence of charges 


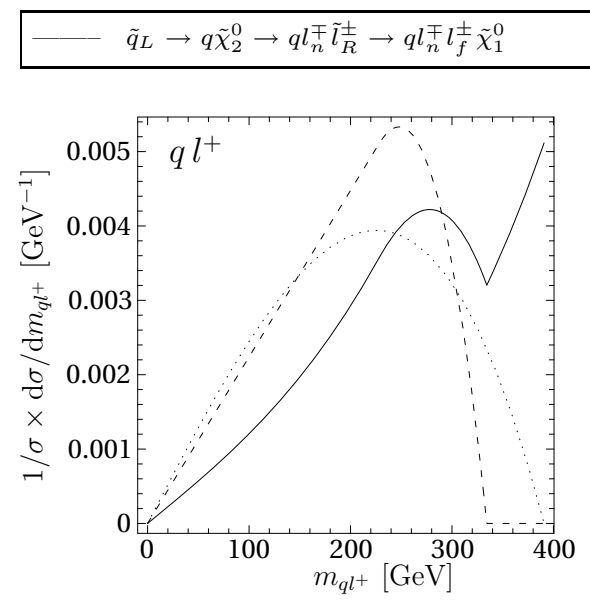

$$
---\tilde{q}_{L} \rightarrow q \tilde{\chi}_{D 2}^{c 0} \rightarrow q l_{n}^{-} \tilde{l}_{R}^{+} \rightarrow q l_{n}^{-} l_{f}^{+} \tilde{\chi}_{D 1}^{c 0}
$$

$\cdots \quad \tilde{q}_{L}^{*} \rightarrow \bar{q} \tilde{\chi}_{D 2}^{0} \rightarrow \bar{q} l_{n}^{+} \tilde{l}_{R}^{-} \rightarrow \bar{q} l_{n}^{+} l_{f}^{-} \tilde{\chi}_{D 1}^{0}$
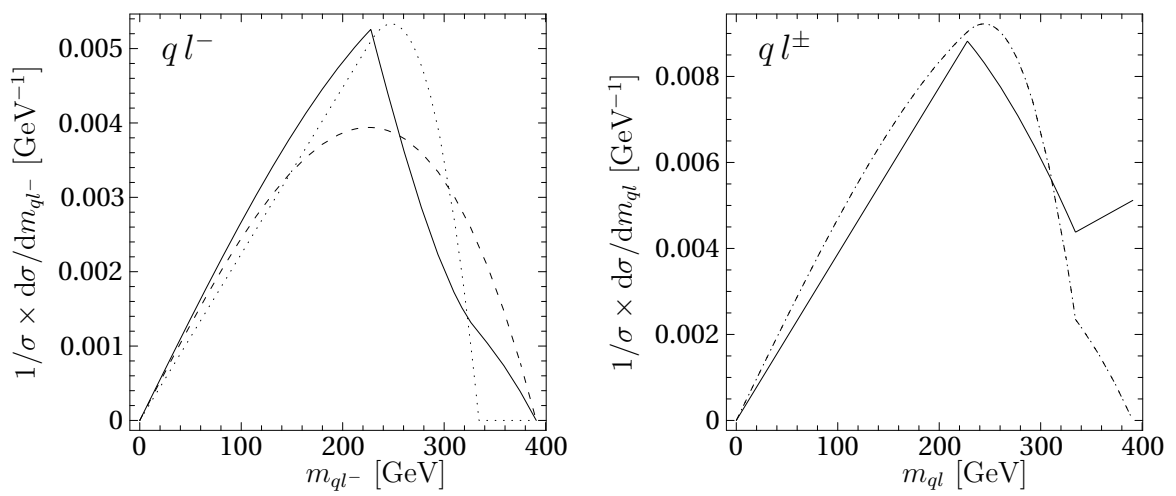

FIG. 6: $q l$ invariant mass distributions for squark decay chains involving Majorana or Dirac neutralinos. The masses have been taken from the SPS1a' scenario [31].

in leptonic decay modes is thus predicted. The squark decay generates, together with the quark, an antineutralino $\tilde{\chi}_{D 2}^{c 0}$, the antineutralino in turn decays to a lepton $l^{-}$and an antislepton $\tilde{l}_{R}^{+}$which finally decays into an antilepton $l^{+}$:

$$
\tilde{q}_{L} \rightarrow q \tilde{\chi}_{D 2}^{c 0} \rightarrow q l_{n}^{-} \tilde{l}_{R}^{+} \rightarrow q l_{n}^{-} l_{f}^{+} \tilde{\chi}_{D 1}^{c 0}
$$

In other words, only one of the two possibilities available in the Majorana theory can be realized in the Dirac theory.

Following the calculations of Ref. [30] we derive the $q l^{-}$and $q l^{+}$distributions as shown in Fig. 6 for the decay chains in Eqs. (4.13) and (4.15). In order to understand these figures, note first that for the given choice of superparticle masses the endpoint of the $q l_{n}$ invariant mass distribution is larger than that for the $q l_{f}$ distribution. Comparison of the solid curves in the first two frames clearly shows that, for $\tilde{q}_{L}$ decay, the $q l^{+}$distribution is significantly harder than the $q l^{-}$distribution; recall that this is true for both the near and far lepton. Turning to the Dirac scenario, we saw that the $l^{+}$from $\tilde{q}_{L}$ decay has to be the far lepton; the dashed curve in the first frame therefore cuts off at the lower $q l_{f}$ endpoint. Note that this distribution is indeed quite hard, i.e. it peaks fairly close to this endpoint. In contrast, the dashed curve in the second frame shows the distribution of the near lepton in $\tilde{q}_{L}$ decay. Since this lepton is negatively charged, the above discussion leads us to expect this distribution to be relatively soft, and indeed it peaks well below its endpoint.

As demonstrated in the figure, the invariant mass distributions are markedly different for the Dirac cascade scenario compared to the Majorana cascade scenario. Though the sensitivity is reduced to some extent, this is true even when the charge of the lepton is undetermined, as a result of the polarization of the $\tilde{\chi}_{2}^{0}$ stemming from the squark decay. Quite generally, the charge conjugated process

$$
\tilde{q}_{L}^{*} \rightarrow \bar{q} \tilde{\chi}_{D 2}^{0} \rightarrow \bar{q} l_{n}^{+} \tilde{l}_{R}^{-} \rightarrow \bar{q} l_{n}^{+} l_{f}^{-} \tilde{\chi}_{D 1}^{0}
$$

leads, by $C P$-invariance, to charge-chirality correlations exactly opposite to Eq. (4.15), so that the $q l^{ \pm}$distribution from $\tilde{q}_{L}$ decays is identical to the $\bar{q} l^{\mp}$ distribution from $\tilde{q}_{L}^{*}$ decays. As a result, the $q l^{ \pm}$spectrum, in contrast to the $q^{+}$and $q l^{-}$spectra, is insensitive to the squark charge so that the analysis of this distribution, not requiring knowledge of the parton distribution functions, is particularly simple. ${ }^{6}$

\footnotetext{
${ }^{6}$ At the LHC one expects more $\tilde{q}_{L}$ than $\tilde{q}_{L}^{*}$ to be produced, i.e. the charge averaging should be done with different weights. This would increase the difference between the two theories even further. Also note that these distributions can be measured directly only for $l=e, \mu$.
} 


\subsection{Electroweak Majorana signatures in $e^{-} e^{-}$collisions}

Polarized electron-electron collisions $[32,33]$ offer a classical and most transparent method for studying the Majorana character of neutralinos:

$$
\begin{aligned}
& e_{L}^{-} e_{L}^{-} \rightarrow \tilde{e}_{L}^{-} \tilde{e}_{L}^{-}, \quad e_{R}^{-} e_{R}^{-} \rightarrow \tilde{e}_{R}^{-} \tilde{e}_{R}^{-} \\
& e_{L}^{-} e_{R}^{-} \rightarrow \tilde{e}_{L}^{-} \tilde{e}_{R}^{-} .
\end{aligned}
$$

All three processes are activated in Majorana theories while, in analogy to $q q$ scattering, the equal-helicity amplitudes vanish for Dirac neutralino exchange. Electron beams can be polarized at linear colliders to nearly $100 \%$ and, as a minor idealization, we will assume complete polarization for the sake of clarity in the following analysis [corrections to this assumption can trivially be implemented].

In the hybrid theory on which we have based the detailed analyses, the scattering amplitudes can be written as:

$$
\begin{aligned}
& A\left[e_{L}^{-} e_{L}^{-} \rightarrow \tilde{e}_{L}^{-} \tilde{e}_{L}^{-}\right]=-2 e^{2}\left[\mathcal{M}_{L L}(s, t)+\mathcal{M}_{L L}(s, u)\right], \\
& A\left[e_{R}^{-} e_{R}^{-} \rightarrow \tilde{e}_{R}^{-} \tilde{e}_{R}^{-}\right]=2 e^{2}\left[\mathcal{M}_{R R}^{*}(s, t)+\mathcal{M}_{R R}^{*}(s, u)\right], \\
& A\left[e_{L}^{-} e_{R}^{-} \rightarrow \tilde{e}_{L}^{-} \tilde{e}_{R}^{-}\right]=e^{2} \lambda^{1 / 2} \sin \theta \mathcal{D}_{L R}(s, t) .
\end{aligned}
$$

Here $\theta$ is the scattering angle, and the dimensionless neutralino functions $\mathcal{M}_{a b}$ and $\mathcal{D}_{a b}(a, b=L, R)$ are defined by

$$
\begin{aligned}
& \mathcal{M}_{a b}(s, t(u))=\sum_{k=1}^{6} \frac{m_{\tilde{\chi}_{k}^{0}}}{\sqrt{s}} \mathcal{V}_{a k} \mathcal{V}_{b k} D_{k t(u)}, \\
& \mathcal{D}_{a b}(s, t(u))=\sum_{k=1}^{6} \mathcal{V}_{a k} \mathcal{V}_{b k}^{*} D_{k t(u)},
\end{aligned}
$$

They are determined by the $t(u)$-channel neutralino propagators $D_{k t(u)}=s /\left(t(u)-m_{\tilde{\chi}_{k}^{0}}^{2}\right)$ and the effective mixing coefficients

$$
\begin{aligned}
& \mathcal{V}_{L k}=\mathcal{N}_{2 k} / 2 c_{W}+\mathcal{N}_{4 k} / 2 s_{W}, \\
& \mathcal{V}_{R k}=\mathcal{N}_{2 k} / c_{W} .
\end{aligned}
$$

The neutralino mixing matrix $\mathcal{N}$ diagonalizes the neutralino mass matrix as $\mathcal{N}^{T} \mathcal{M}_{n} \mathcal{N}=\operatorname{diag}\left(m_{\tilde{\chi}_{1}^{0}}, \ldots, m_{\tilde{\chi}_{6}^{0}}\right)$. The differential cross sections,

$$
\begin{aligned}
\frac{\mathrm{d} \sigma_{L L}}{\mathrm{~d} \cos \theta} & =\frac{\pi \alpha^{2}}{4 s} \lambda^{1 / 2}\left|\mathcal{M}_{L L}(s, t)+\mathcal{M}_{L L}(s, u)\right|^{2}, \\
\frac{\mathrm{d} \sigma_{R R}}{\mathrm{~d} \cos \theta} & =\frac{\pi \alpha^{2}}{4 s} \lambda^{1 / 2}\left|\mathcal{M}_{R R}(s, t)+\mathcal{M}_{R R}(s, u)\right|^{2}, \\
\frac{\mathrm{d} \sigma_{L R}}{\mathrm{~d} \cos \theta} & =\frac{\pi \alpha^{2}}{4 s} \lambda^{3 / 2} \sin ^{2} \theta\left|\mathcal{D}_{L R}(s, t)+\mathcal{D}_{L R}(s, u)\right|^{2},
\end{aligned}
$$

can easily be derived from the scattering amplitudes.

In the standard Majorana limit the expressions reduce to the familiar MSSM form, see e.g. Ref. [34]. The differential cross sections are the same in their form as those in Eq. (4.21), with the $t$ - and $u$-channel exchanges mediated only by the four mass eigenstates $\tilde{\chi}_{1,3,5,6}^{0}$; the other two states $\tilde{\chi}_{2,4}$ are decoupled as $M_{1,2}^{\prime}$ become infinite.

The Dirac limit, on the other hand, is exceptionally simple in the selectron sector in which the Yukawa couplings $\sim m_{e} / v$ can be neglected. The higgsino couplings vanish in this limit and the higgsino admixtures to the $\mathrm{U}(1)$ and $\mathrm{SU}(2)$ gauginos are ineffective. Hence the neutralino system is isomorphic, apart from the $\mathrm{SU}(3)$ symmetry group, to 


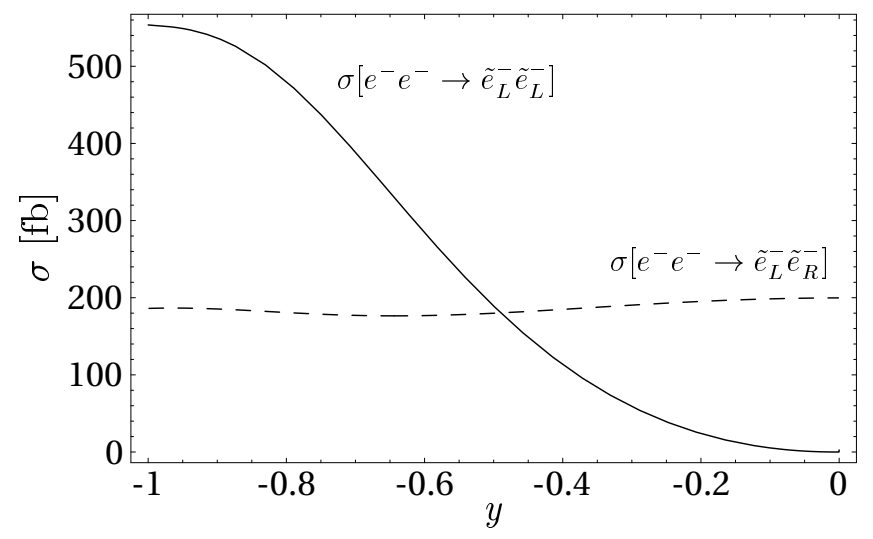

FIG. 7: Partonic cross sections for same-sign selectron production as a functions of the Dirac/Majorana control parameter $y$, for $\sqrt{s}=500 \mathrm{GeV}$ and SPS1a' parameters [31]. Not shown is the cross section for $e^{-} e^{-} \rightarrow \tilde{e}_{R}^{-} \tilde{e}_{R}^{-}$, which, apart from the different normalization, shows a similar behavior as the cross section for $e^{-} e^{-} \rightarrow \tilde{e}_{L}^{-} \tilde{e}_{L}^{-}$.

the gluino system. The differential cross sections in the Dirac limit with the gaugino and higgsino mixing neglected greatly simplify to

$$
\begin{aligned}
\frac{\mathrm{d} \sigma_{L L}}{\mathrm{~d} \cos \theta} & =\frac{\mathrm{d} \sigma_{R R}}{\mathrm{~d} \cos \theta}=0, \\
\frac{\mathrm{d} \sigma_{L R}}{\mathrm{~d} \cos \theta} & =\frac{\mathrm{d} \sigma_{R L}}{\mathrm{~d} \cos \theta}=\frac{\pi \alpha^{2}}{16 c_{W}^{4} s} \lambda^{3 / 2} \sin ^{2} \theta\left(\frac{s}{t-m_{\tilde{\chi}_{1}^{0}}^{2}}+\frac{s}{u-m_{\tilde{\chi}_{1}^{0}}^{2}}\right)^{2} .
\end{aligned}
$$

The two representative cross sections $\sigma_{L L}$ and $\sigma_{L R}$ are shown along the path $\mathcal{P}$, defined analogously to the QCD sector, in Fig. 7. In the figure, gaugino and higgsino mixing induced by electroweak symmetry breaking has been included by diagonalizing the complete mass matrix (2.10) numerically, but the quantitative effect of this mixing is very small.

In Ref. [35] a detailed phenomenological analysis for selectron production in $e^{-} e^{-}$collisions was performed. It was shown that, by using different decay modes of the selectrons, their masses can be reconstructed experimentally, thus allowing a clear distinction between the processes $e^{-} e^{-} \rightarrow \tilde{e}_{R}^{-} \tilde{e}_{R}^{-}, \tilde{e}_{R}^{-} \tilde{e}_{L}^{-}, \tilde{e}_{L}^{-} \tilde{e}_{L}^{-}$. Therefore the Majorana nature of the neutralinos with dominant gaugino component can be tested unambiguously in $e^{-} e^{-}$collisions.

\section{LIKE-SIGN DILEPTONS AND UNLIKE-SIGN DILEPTONS AT THE LHC}

In the previous sections, two methods have been identified for the experimental discrimination between Dirac and Majorana gauginos at the LHC: The correlation between charge and helicity of fermions from Dirac neutralino decays leaves a characteristic imprint on the quark-lepton distributions, as shown in Section 4.2, which cannot be the result of modifications in the sparticle spectrum. Secondly, the production cross sections for squarks and gluinos are different in the two cases, as analyzed in Section 3. In the following it will be shown how this difference can be measured through like-sign and unlike-sign dilepton signals at the LHC. Before describing the detailed phenomenological analysis for the rates of like-sign dilepton events, a general overview of like-sign and unlike-sign dileptons will be given to set the frame for expectations in various channels of the sub-processes. 


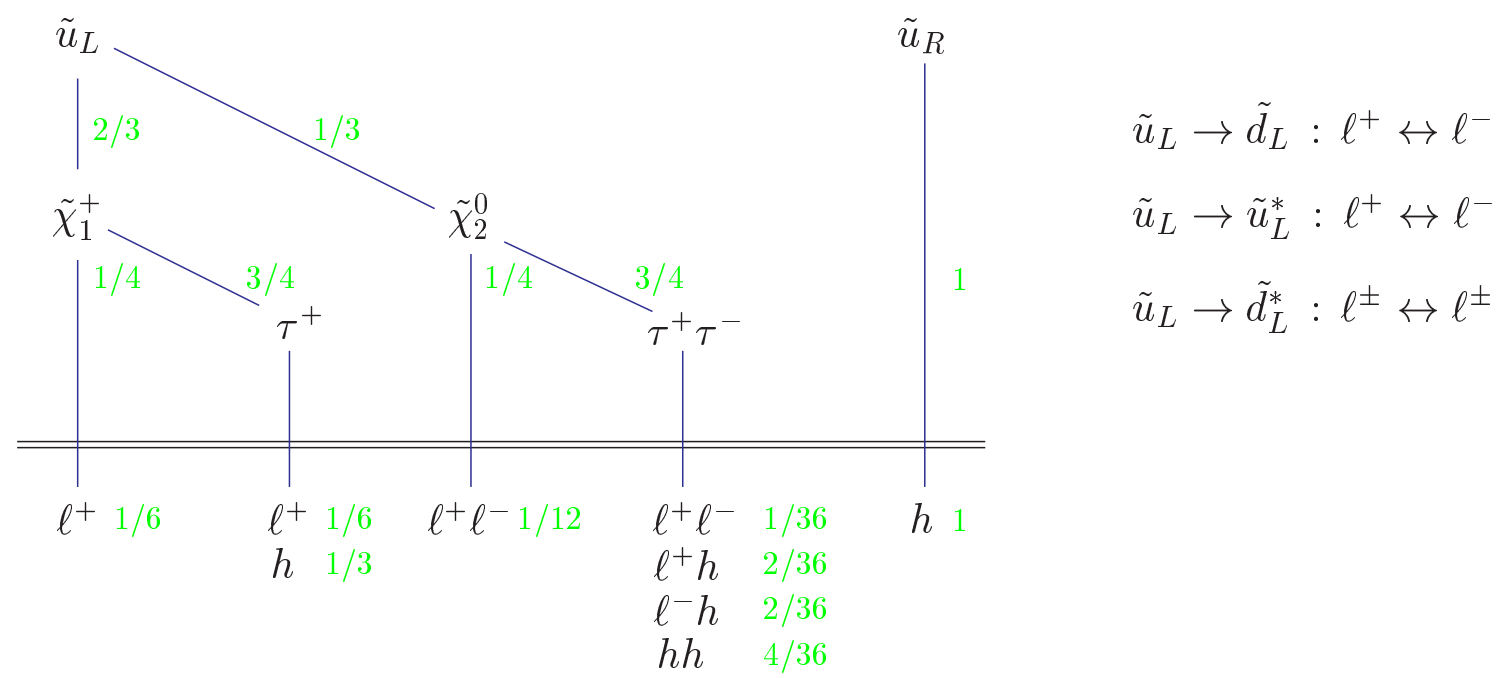

FIG. 8: Sketch of allowed decay chains for L-type and R-type $u$ squarks, $\tilde{u}_{L, R}$, for SPS1a' masses. Here $h$ stands for fully hadronic decay channels without charged leptons, while $\ell$ stands for an electron or muon. The numbers in light green/gray denote approximate branching ratios for the associated decay channels. The decay patterns for the other (anti)squarks can be derived by the replacements given on the right side.

\subsection{A Coarse Picture of Like-Sign and Unlike-Sign Dilepton Channels}

To get a transparent view of channels which allow us to confront the Majorana nature of the gluinos with the Dirac alternative, we will first consider characteristic examples, focusing on the ratio of like-sign dilepton events of different charge and the ratio of like-sign over unlike-sign dileptons. Like-sign lepton pairs can be produced from decays of L-squark pairs mediated by charginos, e.g. for $\tilde{u}$ and $\tilde{d}$-squarks

$$
\begin{aligned}
& \tilde{u}_{L} \rightarrow d \tilde{\chi}_{1}^{+} \rightarrow d l^{+} \nu_{l} \tilde{\chi}_{1}^{0} \\
& \tilde{d}_{L} \rightarrow u \tilde{\chi}_{1}^{-} \rightarrow u l^{-} \bar{\nu}_{l} \tilde{\chi}_{1}^{0},
\end{aligned}
$$

as sketched in Fig. 8. For easy lepton and charge identification, we restrict ourselves to $l=e, \mu \equiv \ell$, or $l=\tau$ with leptonic tau decays $\tau \rightarrow e \nu \bar{\nu}, \mu \nu \bar{\nu}$. Owing to the valence quark distribution in the proton beams, $\ell^{+} \ell^{+}$and $\ell^{-} \ell^{-}$ pairs are not produced in equal numbers in SUSY events. Decay chains with neutralinos, on the other hand,

$$
\tilde{u}_{L} \rightarrow u \tilde{\chi}_{2}^{0} \rightarrow u l^{+} l^{-} \tilde{\chi}_{1}^{0},
$$

lead to predominantly opposite-sign and same-flavor leptons in the final state. [They give only a small contamination to the like-sign dilepton signal when mixed lepton-hadron decays of neutralinos to tau pairs are observed, or, for experimental reasons, when one of the leptons is missed in the detector.] An overview of like-sign and unlike-sign dilepton ratios is presented in Tab. II.

For specifying the decay branching ratios, the reference scenario SPS1a' [31] will be adopted. In this scenario, $\operatorname{BR}\left[\tilde{q}_{L} \rightarrow q^{\prime} \tilde{\chi}_{1}^{ \pm}\right] \approx 2 / 3, \operatorname{BR}\left[\tilde{q}_{L} \rightarrow q \tilde{\chi}_{2}^{0}\right] \approx 1 / 3$ and $\operatorname{BR}\left[\tilde{q}_{R} \rightarrow q \tilde{\chi}_{1}^{0}\right] \approx 1$, which is typical for scenarios with wino-like $\tilde{\chi}_{2}^{0}$ and bino-like $\tilde{\chi}_{1}^{0}$. The charginos $\tilde{\chi}_{1}^{ \pm}$and the neutralino $\tilde{\chi}_{2}^{0}$ decay preferentially to taus with branching ratios $\approx 3 / 4$.

\section{(a) Squark pair production:}

In the Majorana theory the most prominent squark production channels are the subprocesses $u u \rightarrow \tilde{u} \tilde{u}, d d \rightarrow \tilde{d} \tilde{d}$ and $u d \rightarrow \tilde{u} \tilde{d}$, initiated by valence quarks and mediated by gluino exchange. In the Majorana theory, the $\tilde{u}_{L} \tilde{u}_{L}$ and $\tilde{d}_{L} \tilde{d}_{L}$ pair production processes lead to same-sign leptons, whereas opposite-sign dileptons are generated in $\tilde{u}_{L} \tilde{d}_{L}$ events, if both squarks decay into charginos. In both the Dirac and Majorana theory opposite-sign dileptons can 


\begin{tabular}{|c||r|r|r||r|r|r|}
\hline \multicolumn{1}{|c||}{ Process } & \multicolumn{3}{c||}{ Majorana } & \multicolumn{3}{c|}{ Dirac } \\
\cline { 2 - 6 } & $\ell^{+} \ell^{+}$ & $\ell^{-} \ell^{-}$ & $\ell^{+} \ell^{-}$ & $\ell^{+} \ell^{+}$ & $\ell^{-} \ell^{-}$ & $\ell^{+} \ell^{-}$ \\
\hline \hline$u_{L} u_{L} \rightarrow \tilde{u}_{L} \tilde{u}_{L}$ & 49 & 1 & 46 & $\times$ & $\times$ & $\times$ \\
$d_{L} d_{L} \rightarrow \tilde{d}_{L} \tilde{d}_{L}$ & 1 & 49 & 46 & $\times$ & $\times$ & $\times$ \\
$u_{L} d_{L} \rightarrow \tilde{u}_{L} \tilde{d}_{L}$ & 7 & 7 & 82 & $\times$ & $\times$ & $\times$ \\
\hline$u_{L} q_{R} \rightarrow \tilde{u}_{L} \tilde{q}_{R}$ & 0 & 0 & 36 & 0 & 0 & 36 \\
\hline$q_{L} \bar{q}_{L} \rightarrow \tilde{q}_{L} \tilde{q}_{L}^{*}$ & 7 & 7 & 82 & 7 & 7 & 82 \\
$u_{L} \bar{d}_{L} \rightarrow \tilde{u}_{L} \tilde{d}_{L}^{*}$ & 49 & 1 & 46 & 49 & 1 & 46 \\
$u_{L} \bar{q}_{R} \rightarrow \tilde{u}_{L} \tilde{q}_{R}^{*}$ & 0 & 0 & 36 & $\times$ & $\times$ & $\times$ \\
\hline$g u_{L} \rightarrow \tilde{g}_{(D)} \tilde{u}_{L}$ & 14 & 2 & 50 & 14 & 2 & 50 \\
$g \bar{u}_{L} \rightarrow \tilde{g}_{(D)}^{(c)} \tilde{u}_{L}^{*}$ & 2 & 14 & 50 & 2 & 14 & 50 \\
$g q_{R} \rightarrow \tilde{g}_{(D)}^{(c)} \tilde{q}_{R}$ & 0 & 0 & 18 & 0 & 0 & 18 \\
$g \bar{q}_{R} \rightarrow \tilde{g}_{(D)} \tilde{q}_{R}^{*}$ & 0 & 0 & 18 & 0 & 0 & 18 \\
\hline$g g \rightarrow \tilde{g}_{(D)} \tilde{g}_{(D)}^{(c)}$ & 4 & 4 & 34 & 4 & 4 & 34 \\
\hline
\end{tabular}

TABLE II: Approximate relative probabilities of like-sign lepton pairs $\ell^{+} \ell^{+}$and $\ell^{-} \ell^{-}$, and unlike-sign lepton pairs $\ell^{+} \ell^{-}$, separately for characteristic channels $[q=u$ or $d]$; the proper normalization of the probabilities requires dividing all entries by the common denominator $\mathcal{N}_{n}=324$. Probabilities for $\tilde{d}$ processes which can be derived by isospin rotation of $\tilde{u}$ processes are not noted explicitly. Parton processes forbidden in the Dirac theory are marked by the symbol $\times$.

originate from $\tilde{q}_{L} \tilde{q}_{R}$ final states via $\tilde{\chi}_{2}^{0} \rightarrow \ell^{+} \ell^{-}$and hadronic decays of $\tilde{q}_{L}$ and $\tilde{q}_{R}$ squarks, respectively. The following event fractions and ratios

$$
\begin{aligned}
& N\left(\ell^{+} \ell^{+}\right) / N\left(\ell^{-} \ell^{-}\right) \sim 3 \quad \text { (Majorana) } \\
& N\left(\ell^{ \pm} \ell^{ \pm}\right) / N\left(\ell^{+} \ell^{-}\right) \sim 1 / 4
\end{aligned}
$$

and

$$
N\left(\ell^{ \pm} \ell^{ \pm}\right) / N\left(\ell^{+} \ell^{-}\right)=0 \quad(\text { Dirac })
$$

are obtained for $(2 u+d)$ valence partons in the proton.

In both the Majorana and the Dirac theory, squark pairs can also be produced from quark-antiquark scattering and gluon annihilation. The dominant contributions for dileptons come from the processes, $u_{L} \overline{u_{L}} \rightarrow \tilde{u}_{L} \tilde{u}_{L}^{*}, d_{L} \overline{d_{L}} \rightarrow \tilde{d}_{L} \tilde{d}_{L}^{*}$, $u_{L} \overline{d_{L}} \rightarrow \tilde{u}_{L} \tilde{d}_{L}^{*}$ and $d_{L} \overline{u_{L}} \rightarrow \tilde{d}_{L} \tilde{u}_{L}^{*}$. These channels have one valence quark and one sea antiquark in the initial state, so that the cross sections are smaller than the quark-quark cross sections. The channels predict a ratio $N\left(\ell^{+} \ell^{+}\right) / N\left(\ell^{-} \ell^{-}\right) \sim 2$, for an approximate fraction $1 / 4$ of like-sign events within the total dilepton sample. These leading channels are not altered by switching from the Majorana to the Dirac theory.

Channels that are initiated by two sea (anti)quarks are doubly suppressed.

\section{(b) Super-Compton Process:}

Gluinos $\tilde{g}$ decay in the Majorana theory democratically at equal rates to $\tilde{u}, \tilde{u}^{*}$ and $\tilde{d}, \tilde{d}^{*}$ squarks, both L- and R-types, of the first two generations. Therefore the super-Compton process $q g \rightarrow \tilde{q} \tilde{g}$ generates like-sign leptons with a branching ratio that is independent of the squark charge. [Second generation $\tilde{s}, \tilde{c}$ squarks will be included in the subsequent phenomenological analysis.] However, since the super-Compton process is predominantly initiated by valence quarks, positively charged like-sign leptons pairs outnumber negatively charged like-sign pairs by the ratio 
$N\left(\ell^{+} \ell^{+}\right) / N\left(\ell^{-} \ell^{-}\right) \sim 2$. The number of unlike-sign dilepton events is dominant, the ratio $N\left(\ell^{+} \ell^{+}+\ell^{-} \ell^{-}\right) / N\left(\ell^{+} \ell^{-}\right) \sim$ $1 / 4$, due to the additional enhancement of the final states generated by $\tilde{q}_{L} \rightarrow \tilde{\chi}_{2}^{0}$ decays accompanied by non-leptonic $\tilde{q}_{R}$ jet decays.

The picture becomes a bit more subtle when switching to the Dirac theory. L-squarks $\tilde{q}_{L}$ are only produced together with $\tilde{g}_{D}$ gluinos, whereas $\sigma\left[g q \rightarrow \tilde{q}_{L} \tilde{g}_{D}^{c}\right]=0$, see Eq. (3.38). According to Eqs. (3.49) and (3.50), the $\tilde{g}_{D}$ gluinos subsequently decay only into $\tilde{u}_{L}^{*}, \tilde{d}_{L}^{*}$, but not into $\tilde{u}_{L}, \tilde{d}_{L}$. Nevertheless, since gluinos decay democratically to each flavor (anti)-squark for equal masses, the probabilities of the like-sign and unlike-sign lepton pairs are not altered by switching from the Majorana to the Dirac theory. [For $\tan \beta>1, \tilde{d}_{L}$ squarks are slightly heavier than $\tilde{u}_{L}$ squarks, so that the latter are slightly preferred in gluino decays. This small effect has been taken into account in the numerical analysis to be described in the following subsection.]

\section{(c) Gluino Pair-Production:}

Pair production of gluinos in the Majorana theory leads to same-sign L-squark pairs $\left(\tilde{u}_{L} \tilde{u}_{L}, \tilde{u}_{L}^{*} \tilde{u}_{L}^{*}, \tilde{d}_{L} \tilde{d}_{L}, \tilde{d}_{L}^{*} \tilde{d}_{L}^{*}\right.$, $\tilde{u}_{L} \tilde{d}_{L}^{*}$ and $\left.\tilde{u}_{L}^{*} \tilde{d}_{L}\right)$ in half of the cases, which in turn generate same-sign leptons pairs through the chargino decay chain, with the charge ratio $N\left(\ell^{+} \ell^{+}\right) / N\left(\ell^{-} \ell^{-}\right) \sim 1$. In the Dirac theory, only $\tilde{g}_{D} \tilde{g}_{D}^{c}$ gluino pairs are generated and $\tilde{g}_{D}$ decays only into L-antisquarks $\tilde{q}_{L}^{*}$ (and R-squarks $\tilde{q}_{R}$ ) while $\tilde{g}_{D}^{c}$ decays only into L-squarks $\tilde{q}_{L}$ (and R-antisquarks $\left.\tilde{q}_{R}^{*}\right)$. However, as for case (b), owing to the flavor-democratic decays of (Dirac) gluinos the relative rates for like-sign and unlike-sign lepton pairs are unchanged for the Dirac theory compared to the Majorana theory. In other words, contrary to popular belief the frequent occurrence of like-sign dilepton pairs in gluino pair events is not a signal for the Majorana nature of the gluino.

The total production cross section for gluino pairs is roughly twice as large in the Dirac theory compared to the Majorana theory, as a result of the doubling of the physical degrees of freedom of the gluinos.

In summary, the population of like-sign dileptons predicted in the Majorana theory is altered significantly when switching to the Dirac theory, with the suppression of like-sign as well as unlike-sign dileptons in the valence channels being most prominent. Properly weighing the individual channels,

$$
\ell^{+} \ell^{+} / \ell^{-} \ell^{-} / \ell^{+} \ell^{-}=\sum \sigma_{k} f_{k}^{ \pm \pm /+-} / \sum \sigma_{k}
$$

the valence-valence and super-Compton channels generate the leading contributions, of similar size as demonstrated in the next subsection. In addition to the absolute rates for $\ell^{+} \ell^{+}$and $\ell^{-} \ell^{-}$production, it is very useful to tag the large transverse momentum jets in the like-sign dilepton events, since this observation allows us to discriminate between squark and gluino production as the primary hard process $[9,10]$. Thus, detailed analyses of dilepton events can provide powerful discriminants between the Majorana and Dirac nature of the gluinos.

\subsection{A Detailed Analysis of Like-Sign Dileptons in Majorana/Dirac Theories}

Since our numerical analysis of like-sign dileptons follows strictly the report on the measurement of the Yukawa coupling in super-QCD, we will not repeat any of the technical points described comprehensively in Refs. [9, 10]. For representative numerical results, we will adopt the MSSM scenario of this study, which is close to the reference points SPS1a' [31] and the Snowmass point SPS1a [36]. Though the supersymmetry mass spectrum is comparatively light, it is compatible, nevertheless, with analyses of high precision electroweak measurements [37]. Higher supersymmetric masses reduce the production rates and would thus require larger integrated luminosities at the LHC to obtain similar event numbers. We note, however, that the processes initiated purely by valence quarks will drop off most slowly. Heavier spectra thus mean less "pollution" of the SUSY dilepton sample by events with gluinos in the final state; we saw above that ratios of dilepton final states in these gluino events are identical in the Dirac and Majorana theories. Increasing the sparticle masses should therefore reduce the number of events needed to cleanly distinguish between 


\begin{tabular}{|l||r|r||r|r||r|r|}
\hline \multirow{2}{*}{ Process } & \multicolumn{2}{|c||}{ Majorana } & \multicolumn{2}{|c||}{ Dirac } & \multicolumn{2}{|c|}{$N\left(\ell^{+} \ell^{+}\right) / N\left(\ell^{-} \ell^{-}\right)$} \\
\cline { 2 - 7 } & Total cross-section & With BRs and cuts & Total cross-section & With BRs and cuts & Majorana & Dirac \\
\hline \hline$\sigma\left[\tilde{q}_{L} \tilde{q}_{L}^{(\prime)}\right]$ & $2.1 \mathrm{pb}$ & $0.1 \mathrm{fb}$ & 0 & 0 & 2.5 & - \\
$\sigma\left[\tilde{q}_{L} \tilde{q}_{L}^{(\prime) *}\right]$ & $3.1 \mathrm{fb}$ & $1.4 \mathrm{pb}$ & $3.1 \mathrm{fb}$ & 1.4 & 1.4 \\
$\sigma\left[\tilde{q}_{L} \tilde{g}_{(D)}\right]$ & $1.4 \mathrm{pb}$ & $7.0 \mathrm{pb}$ & $7.6 \mathrm{fb}$ & 1.5 & 1.5 \\
$\sigma\left[\tilde{g}_{(D)} \tilde{g}_{(D)}^{(c)}\right]$ & $7.0 \mathrm{pb}$ & $7.4 \mathrm{fb}$ & $3.2 \mathrm{fb}$ & 1.0 & 1.0 \\
\hline$\sigma[\mathrm{SM}]$ & $3.2 \mathrm{pb}$ & $1.4 \mathrm{fb}$ & $<00 \mathrm{pb}$ & $<0.6 \mathrm{fb}$ & 1.0 \\
\hline
\end{tabular}

TABLE III: Signal and background cross-sections before and after including branching ratios (BRs) and applying the cuts of Ref. [10]. The numbers always include also the charge conjugate of the processes in the first column.

the two theories.

The masses and branching ratios for squarks, gluinos and charginos/neutralinos of SPS1a ${ }^{\prime}$ are tabulated in Ref. [9, 10]. In this scenario, gluinos are heavier than squarks so that they decay via $\tilde{g} \rightarrow \tilde{q} \bar{q}, \tilde{q}^{*} q$. The branching ratios involving charginos and neutralinos are not altered when switching from the Majorana to the Dirac theory, except for the charge-helicity correlations discussed in Section 4.2, which however do not matter for this analysis. ${ }^{7}$

Based on the parton cross sections derived in the preceding sections, the theoretical predictions for the $p p$ cross sections at the LHC are summarized in Tab. III. The values are given, in the Majorana as well as the Dirac theory, for the relevant squark and gluino channels. Parallel to Ref. [10] a set of cuts has been applied to fight the huge background cross section from the Standard Model processes: at least two jets with $p_{\mathrm{T}, \mathrm{j}}>200 \mathrm{GeV}$, missing transverse energy $\mathbb{E}_{\mathrm{T}}>300 \mathrm{GeV}$, exactly two isolated same-sign leptons $\ell=e, \mu$ with $p_{\mathrm{T}, \ell}>7 \mathrm{GeV}$, and a bottom-flavor veto. After applying the cuts, this SM background is suppressed to a level of $5 \%$. Also shown in the table is the ratio of reconstructed positively and negatively charged lepton pairs, $N\left(\ell^{+} \ell^{+}\right) / N\left(\ell^{-} \ell^{-}\right)$, for each of the production channels. As a result of the more realistic simulation, the values for $N\left(\ell^{+} \ell^{+}\right) / N\left(\ell^{-} \ell^{-}\right)$are washed out compared to the naive estimates of the previous subsection.

For an integrated luminosity of $\int \mathcal{L}=300 \mathrm{fb}^{-1}$ at the LHC one obtains the following event numbers for the final states with $\ell^{+} \ell^{+}$and $\ell^{-} \ell^{-}$,

$$
\begin{aligned}
& \text { Majorana: } \quad N\left(\ell^{+} \ell^{+}\right)=3,500 \\
& \text { Dirac: } \quad N\left(\ell^{+} \ell^{+}\right)=2,400 \\
& N\left(\ell^{-} \ell^{-}\right)=2,100 \\
& N\left(\ell^{+} \ell^{+}\right) / N\left(\ell^{-} \ell^{-}\right)=1.66 \\
& N\left(\ell^{-} \ell^{-}\right)=1,800 \\
& N\left(\ell^{+} \ell^{+}\right) / N\left(\ell^{-} \ell^{-}\right)=1.33 \text {. }
\end{aligned}
$$

It is advantageous to focus on cross section ratios only, so that uncertainties for the total luminosity and the branching ratios in the decay chains Eq. (5.1) cancel out. From the measurement of the ratio $N\left(\ell^{+} \ell^{+}\right) / N\left(\ell^{-} \ell^{-}\right)$the Dirac theory, in comparison to the Majorana theory, can be rejected with a statistical significance of more than $7 \sigma$.

However, systematic error sources are important and need to be taken into account. Large sources for systematic uncertainties are the measurement of the squark and gluino masses, the proton parton distribution functions (PDFs) and missing next-to-next-to-leading order radiative corrections for the production cross sections. Following Ref. [10], we assume $\delta m_{\tilde{g}}=12 \mathrm{GeV}$ and $\delta m_{\tilde{q}}=10 \mathrm{GeV}$ and derive the error from higher order corrections from the scale dependence of the next-to-leading order result [22]. For the PDFs we expect that the current uncertainty will be improved by a factor of two due to the final HERA analyses for the gluon PDF, and Tevatron and LHC data for the quark PDFs. Including these systematic errors, the significance is reduced to a level of about $2 \sigma$.

\footnotetext{
7 The simulation has been performed using PYTHIA [38], which does not keep track of the polarization of decaying neutralinos. Since we use very mild cuts on the charged leptons, these polarization effects should not change the event numbers significantly.
} 

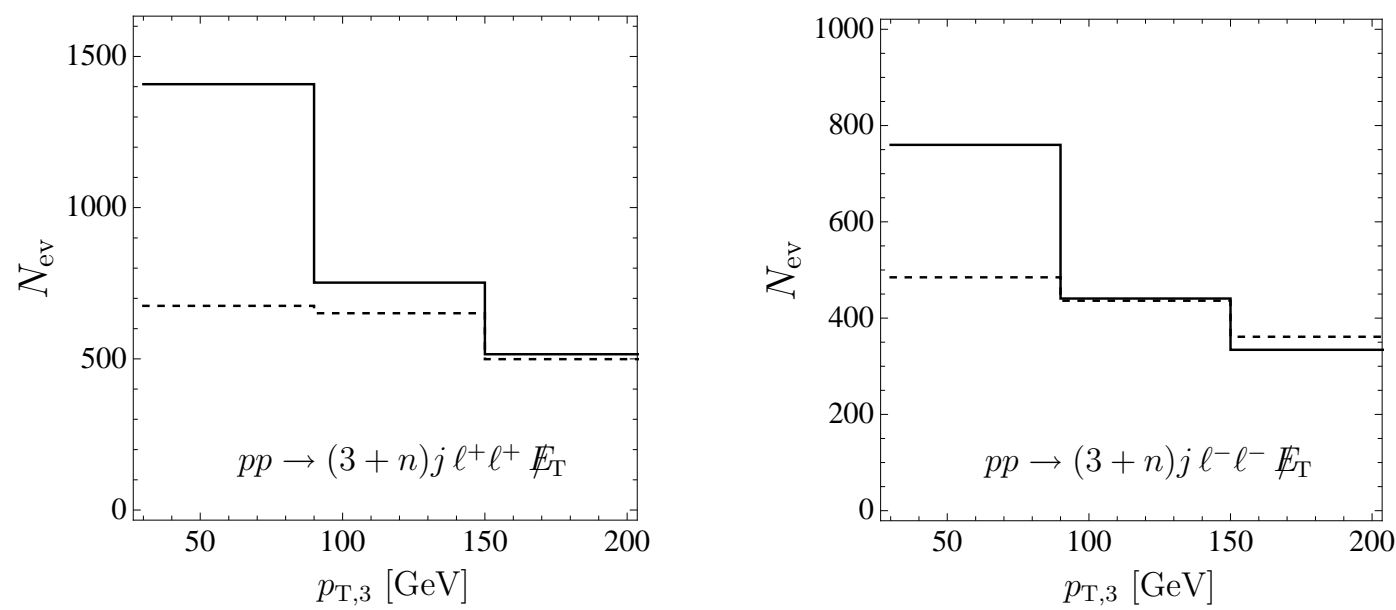

FIG. 9: Distribution of the transverse momentum of the third jet for the $\ell^{+} \ell^{+}$(left) and the $\ell^{-} \ell^{-}$(right) signal stemming from squark and/or gluino production, for Majorana theory (solid) and Dirac theory (dashed). The plots show the distributions in three bins, for SPS1a' masses.

Fortunately the result can be improved considerably by considering the distribution of the transverse momentum of the third hardest jet, $p_{\mathrm{T}, 3}$ in the signal events, which accentuates gluino decays. Due to the extra jet from the gluino decay $\tilde{g} \rightarrow \tilde{q} \bar{q}, \tilde{q}^{*} q$, this distribution is sensitive to the relative contributions from squark pair production, squarkgluino production, and gluino pair production. As shown in Fig. 9, in the Majorana theory the $p_{\mathrm{T}, 3 \text {-distribution is }}$ peaked at low values of $p_{\mathrm{T}, 3}$, as a result of the sizable contribution from $\tilde{q}_{L} \tilde{q}_{L}$ production. On the other hand, the Dirac theory predicts a relatively larger signal from the squark-gluino super-Compton process compared to squark pair production. The gluino decay leads to a hard third jet, so that the $p_{\mathrm{T}, 3}$-distribution falls off more slowly towards high momenta.

Dividing the $p_{\mathrm{T}, 3}$-spectrum into 3 bins in the range $p_{\mathrm{T}, 3} \in[30,200] \mathrm{GeV}$, a fit to the distributions for the $\ell^{+} \ell^{+}$ and $\ell^{-} \ell^{-}$final states allows a statistical discrimination between the Majorana and Dirac theory with 11.3 standard deviations (for $\int \mathcal{L}=300 \mathrm{fb}^{-1}$ ). Taking into account systematic errors as above, we find that the Dirac theory can be separated from the Majorana theory by more than $10.7 \sigma$.

Finally, we comment on a subtle issue: If the Yukawa coupling between gluinos, squarks and quarks is treated as an unknown parameter, as in Ref. $[9,10]$, one may worry that a non-standard value of the Yukawa coupling mimics the effect of the Dirac theory at the LHC. We have analyzed this problem by repeating the fit to the $p_{\mathrm{T}, 3}$-spectrum with the Yukawa coupling as a free parameter. We have also included a total cross section measurement in this fit, with a conservative error of $30 \%$. It turns out that with a free-floating Yukawa coupling the Majorana and Dirac theories can be distinguished with a reduced statistical significance of $4.7 \sigma$ (4.5 $\sigma$ including systematics). Thus, with a medium significance, the Yukawa coupling and the Majorana/Dirac nature of the gluinos can be determined simultaneously and independently.

\section{SUMMARY}

If supersymmetry is realized in nature at low energies, the next steps after the discovery of supersymmetric particles will be the measurement of their properties. While the measurements of masses, spins and Yukawa couplings at the LHC has been discussed in earlier reports [9, 10, 29, 30, 39], we have focused here on studies of the Majorana nature of gluinos [and in a restricted form on neutralinos which will be treated in depth in a later investigation].

The parallelism between self-conjugate neutral gauge bosons and their fermionic supersymmetric partners induces the Majorana nature of these particles in the minimal formulation of the theory. Nevertheless, experimental tests of 
the Majorana character would provide non-trivial insight into the potential realization of supersymmetry in nature, since extended supersymmetric models can include Dirac gauginos. $\mathrm{N}=2$ supersymmetry provides a solid theoretical basis for formulating such a testing ground. Since the fermionic degrees of freedom are doubled in the gauge sector, the ensuing two Majorana fields can be joined to a single Dirac field if the masses are chosen identical. Moreover, a continuous path could be designed connecting the original MSSM N=1 Majorana theory and the N=2 Dirac theory by variation of mass parameters. The MSSM corresponds in this frame to a parameter space point in which one of the $\mathrm{N}=2$ mass parameters is shifted to infinity, leading to the decoupling of the additional gaugino states. For equal mass parameters, on the other side, the Dirac theory emerges in a natural way.

It is interesting to note that the transition from the Majorana to the Dirac theory is smooth, suggesting the notion of a near-Dirac field in the approach to the Dirac limit. This notion proves very useful in the analysis of the two theories.

There are several methods to investigate the Majorana nature of gluinos. In the original form, decays to heavy stop/top quarks are exploited [12] to study that the final state in the fermion decay $\tilde{g} \rightarrow \tilde{t} \bar{t}+\tilde{t}^{*} t$ is self-conjugate. In this report we have explored an alternative by studying the nature of $t$-channel exchanged gluinos. While the cross section for the scattering processes with equal-chirality quarks $q_{L} q_{L} \rightarrow \tilde{q}_{L} \tilde{q}_{L}$ is non-zero in the Majorana theory, it vanishes in the Dirac theory. Likewise for two R-chiralities. However, note that two unlike-chirality quarks can generate squarks also in the Dirac theory. L-squarks in the final state can be tagged by measuring the lepton charges in their chargino decay modes. Owing to the dominance of $u$-quarks over $d$-quarks in the proton, the Majorana theory predicts large rates of like-sign dilepton final states from squark pair production with an excess of positively charged leptons while they are absent, apart from a small number of remnant channels, in the Dirac theory. In a realistic analysis one has to include gluino production processes which can also feed the like-sign dilepton signal but can be discriminated by extra jet emission from the gluino decays. Conclusio generalis, the Majorana theory can be discriminated from the Dirac theory using like-sign dilepton events at the level of more than $10 \sigma$.

In this analysis we focussed on a scenario where gluinos are somewhat heavier than first and second generation squarks. If gluinos are much lighter, most squarks will decay into gluinos rather than into neutralinos and charginos. In this case one expects, in toto, approximately equal $\ell^{+} \ell^{+}$and $\ell^{-} \ell^{-}$events in both the Majorana and the Dirac theory. However, the dominant process will then be gluino pair production, which has a two times larger cross section in the Dirac theory. In addition, left/right-chiral correlations among top or bottom quark pairs in gluino-pair decays are different in the Majorana and Dirac theory and they generate different experimental signatures. While we did not perform a detailed analysis of such a scenario, we expect that the two discriminants should allow a clean separation of the Majorana and Dirac theories also in this case.

Similar analyses can also be designed for electroweak neutralinos. Some of these tests can be performed at the LHC while other very clean reactions, like $e^{-} e^{-} \rightarrow \tilde{e}^{-} \tilde{e}^{-}$, can be carried out at TeV linear colliders. The results of these investigations will be presented in a sequel to this report.

\section{Acknowledgments}

We are grateful to A. M. Cooper-Sarkar, A. Glazov, T. Hebbeker, and S. Lammel for communications on various experimental aspects of this study. Particular thanks go to J. Kalinowski for the critical reading of the manuscript. Special thanks go to M. M. Mühlleitner and P. Skands for clarifying issues on the branching ratios of gluino decays. The work by SYC was supported in part by the Korea Research Foundation Grant funded by the Korean Government (MOERHRD, Basic Research Promotion Fund) (KRF-2007-521-C00065) and in part by KOSEF through CHEP at Kyungpook National University. The work of MD was partially supported by Bundesministerium für Bildung und Forschung under contract no. 05HT6PDA, and partially by the Marie Curie Training Research Networks "Uni- 
verseNet" under contract no. MRTN-CT-2006-035863, "ForcesUniverse" under contract no. MRTN-CT-2004-005104, as well as "The Quest for Unification" under contract no. MRTN-CT-2004-503369. Work at ANL is supported in part by the US DOE, Division of HEP, Contract DE-AC-02-06CH11357. PMZ is grateful to the Inst. Theor. Phys. E for the warm hospitality extended to him at RWTH Aachen.

[1] Yu. A. Golfand and E. P. Likhtman, JETP Lett. 13 (1971) 3214; J. Wess and B. Zumino, Nucl. Phys. B 70 (1974) 39.

[2] H. P. Nilles, Phys. Rept. 110 (1984) 1; H. E. Haber and G. L. Kane, Phys. Rept. 117 (1985) 75.

[3] M. Drees, R. Godbole and P. Roy, "Theory and phenomenology of sparticles: An account of four-dimensional N=1 supersymmetry in high energy physics," Hackensack, USA: World Scientific (2004) 555 p; P. Binetruy, "Supersymmetry: Theory, experiment and cosmology," Oxford, UK: Oxford Univ. Pr. (2006) 520 p; J. Wess and J. Bagger, Princeton, USA: Univ. Pr. (1992) 259 p.

[4] K. Benakli and C. Moura, in M. M. Nojiri et al., arXiv:0802.3672 [hep-ph].

[5] P. Fayet, Nucl. Phys. B 113 (1976) 135; L. Álvarez-Gaumé and S. F. Hassan, Fortsch. Phys. 45 (1997) 159 [arXiv:hepth/9701069].

[6] See, e.g., S. M. Bilenky and S. T. Petcov, Rev. Mod. Phys. 59 (1987) 671 [Erratum-ibid. 61 (1989) 169]; R. N. Mohapatra and P. B. Pal, World Sci. Lect. Notes Phys. 72 (2004) 1.

[7] A. Airapetian et al., ATLAS Detector and physics performance technical design report, Vol. 1, ATLAS-TDR-14, CERN/LHCC-99-14; G.L. Bayatian et al., CMS technical design report, volume II: Physics performance, J. Phys. G 34, 995 (2007).

[8] E. Accomando et al., Phys. Rept. 299 (1998) 1 [arXiv:hep-ph/9705442]; J. A. Aguilar-Saavedra et al. [ECFA/DESY LC Physics Working Group], TESLA Technical Design Report Part III: Physics at an e+e- Linear Collider, arXiv:hepph/0106315; J. Brau et al. [ILC Collaboration], ILC Reference Design Report, Vol. 1 - Executive Summary, arXiv:0712.1950 [physics.acc-ph]; A. Djouadi et al., ILC Reference Design Report, Vol.2, arXiv:0709.1893 [hep-ph]; E. Accomando et al. [CLIC Physics Working Group], Physics at the CLIC multi-TeV linear collider, arXiv:hep-ph/0412251.

[9] A. Freitas and P. Z. Skands, JHEP 0609 (2006) 043; see also A. Brandenburg, M. Maniatis, M. M. Weber and P. M. Zerwas, arXiv:0806.3875 [hep-ph].

[10] A. Freitas, P. Z. Skands, M. Spira and P. M. Zerwas, JHEP 0707 (2007) 025, [arXiv:hep-ph/0703160].

[11] M. M. Nojiri and M. Takeuchi, Phys. Rev. D 76 (2007) 015009 [arXiv:hep-ph/0701190].

[12] R. M. Barnett, J. F. Gunion and H. E. Haber, Phys. Lett. B 315 (1993) 349 [arXiv:hep-ph/9306204]; S. Kraml and A. R. Raklev, Phys. Rev. D 73 (2006) 075002 [arXiv:hep-ph/0512284]; A. Alves, O. Eboli and T. Plehn, Phys. Rev. D 74 (2006) 095010 [arXiv:hep-ph/0605067].

[13] A. Abulencia et al., The CDF Collaboration, Phys. Rev. Lett. 96 (2006) 171802; and http://www-cdf.fnal.gov/physics/ exotic/r2a/20080410.bbmet \-gluinosbottom/.

[14] J. Alwall, D. Rainwater and T. Plehn, Phys. Rev. D 76 (2007) 055006 [arXiv:0706.0536 [hep-ph]].

[15] S. Y. Choi and Y. G. Kim, Phys. Rev. D 69 (2004) 015011 [arXiv:hep-ph/0311037]; S. Y. Choi, B. C. Chung, J. Kalinowski, Y. G. Kim and K. Rolbiecki, Eur. Phys. J. C 46 (2006) 511 [arXiv:hep-ph/0504122].

[16] P. J. Fox, A. E. Nelson and N. Weiner, JHEP 0208, 035 (2002) [arXiv:hep-ph/0206096].

[17] I. Antoniadis, K. Benakli, A. Delgado and M. Quiros, CERN-PH-TH-2006-188 [arXiv:hep-ph/0610265].

[18] Z. Chacko, P. J. Fox and H. Murayama, Nucl. Phys. B 706 (2005) 53 [arXiv:hep-ph/0406142].

[19] Y. Nomura, D. Poland and B. Tweedie, Nucl. Phys. B 745, 29 (2006) [arXiv:hep-ph/0509243].

[20] L. J. Hall and L. Randall, Nucl. Phys. B 352, 289 (1991); G. D. Kribs, E. Poppitz and N. Weiner, arXiv:0712.2039 [hep-ph].

[21] S. Y. Choi, H. E. Haber, J. Kalinowski and P. M. Zerwas, Nucl. Phys. B 778 (2007) 85 [arXiv:hep-ph/0612218].

[22] W. Beenakker, R. Höpker, M. Spira and P. M. Zerwas, Nucl. Phys. B 492 (1997) 51 [arXiv:hep-ph/9610490].

[23] P. R. Harrison and C. H. Llewellyn Smith, Nucl. Phys. B 213 (1983) 223 [Erratum-ibid. B 223 (1983) 542]; S. Dawson, E. Eichten and C. Quigg, Phys. Rev. D 31 (1985) 1581.

[24] A. Kulesza and L. Motyka, arXiv:0807.2405 [hep-ph].

[25] S. Bornhauser, M. Drees, H. K. Dreiner and J. S. Kim, Phys. Rev. D 76, 095020 (2007) [arXiv:0709.2544 [hep-ph]].

[26] W. Hollik and E. Mirabella, arXiv:0806.1433 [hep-ph].

[27] W. Beenakker, R. Höpker and P. M. Zerwas, Phys. Lett. B 378 (1996) 159 [arXiv:hep-ph/9602378]. 
[28] M. Mühlleitner, A. Djouadi and Y. Mambrini, Comput. Phys. Commun. 168 (2005) 46 [arXiv:hep-ph/0311167].

[29] A. J. Barr, Phys. Lett. B 596 (2004) 205 [arXiv:hep-ph/0405052].

[30] J. M. Smillie and B. R. Webber, JHEP 0510 (2005) 069 [arXiv:hep-ph/0507170]; D. J. Miller, P. Osland and A. R. Raklev, JHEP 0603 (2006) 034 [arXiv:hep-ph/0510356].

[31] J. A. Aguilar-Saavedra et al., Eur. Phys. J. C 46 (2006) 43 [arXiv:hep-ph/0511344].

[32] W. Y. Keung and L. Littenberg, Phys. Rev. D 28, 1067 (1983).

[33] G. A. Moortgat-Pick et al., Phys. Rept. 460 (2008) 131 [arXiv:hep-ph/0507011].

[34] A. Freitas, A. von Manteuffel and P. M. Zerwas, Eur. Phys. J. C 34 (2004) 487 [arXiv:hep-ph/0310182].

[35] J. A. Aguilar-Saavedra and A. M. Teixeira, Nucl. Phys. B 675 (2003) 70 [arXiv:hep-ph/0307001].

[36] B. C. Allanach et al., "The Snowmass points and slopes: Benchmarks for SUSY searches," in Proc. of the APS/DPF/DPB Summer Study on the Future of Particle Physics, Snowmass (Colorado) 2001, and Eur. Phys. J. C 25 (2002) 113 [arXiv:hep$\mathrm{ph} / 0202233]$.

[37] J. R. Ellis, S. Heinemeyer, K. A. Olive, A. M. Weber and G. Weiglein, JHEP 0708 (2007) 083 [arXiv:0706.0652 [hep-ph]].

[38] T. Sjöstrand, S. Mrenna and P. Skands, JHEP 0605, 026 (2006) [arXiv:hep-ph/0603175].

[39] I. Hinchliffe, F. E. Paige, M. D. Shapiro, J. Söderqvist and W. Yao, Phys. Rev. D 55, 5520 (1997) [arXiv:hep-ph/9610544]; C. G. Lester and D. J. Summers, Phys. Lett. B 463, 99 (1999) [arXiv:hep-ph/9906349]; G. Weiglein et al. [LHC/LC Study Group], Phys. Rept. 426 (2006) 47 [arXiv:hep-ph/0410364]. 